\title{
Coronal properties of the EQ Pegasi binary system
}

\author{
C. Liefke ${ }^{1}$, J.-U. Ness ${ }^{2}$, J. H. M. M. Schmitt ${ }^{1}$, and A. Maggio ${ }^{3}$ \\ ${ }^{1}$ Hamburger Sternwarte, Universät Hamburg, Gojenbergsweg 112, 21029 Hamburg, Germany \\ e-mail: cliefke@hs .uni-hamburg.de \\ 2 Arizona State University, School of Earth and Space Exploration, ASU, PO Box 871404, Tempe, AZ 85287, USA \\ 3 INAF - Osservatorio Astronomico di Palermo, Piazza del Parlamento 1, 90134 Palermo, Italy
}

Received 25 April 2008 / Accepted 20 September 2008

\section{ABSTRACT}

\begin{abstract}
Context. The activity indicators of $\mathrm{M}$ dwarfs are distinctly different for early and late types. The coronae of early $\mathrm{M}$ dwarfs display high X-ray luminosities and temperatures, a pronounced inverse FIP effect, and frequent flaring to the extent that no quiescent level can be defined in many cases. For late M dwarfs, fewer but more violent flares have been observed, and the quiescent X-ray luminosity is much lower.

Aims. To probe the relationship between coronal properties with spectral type of active M dwarfs, we analyze the M3.5 and M4.5 components of the EQ Peg binary system in comparison with other active M dwarfs of spectral types M0.5 to M5.5.

Methods. We investigate the timing behavior of both components of the EQ Peg system, reconstruct their differential emission measure, and investigate the coronal abundance ratios based on emission-measure independent line ratios from their Chandra HETGS spectra. Finally we test for density variations in different states of activity.

Results. The X-ray luminosity of EQ Peg A (M3.5) is by a factor of 6-10 brighter than that of EQ Peg B (M4.5). Like most other active M dwarfs, the EQ Peg system shows an inverse FIP effect. The abundances of both components are consistent within the errors; however, there seems to be a tendency toward the inverse FIP effect being less pronounced in the less active EQ Peg B when comparing the quiescent state of the two stars. This trend is supported by our comparison with other M dwarfs.

Conclusions. As the X-ray luminosity decreases with later spectral type, so do coronal temperatures and flare rate. The amplitude of the observed abundance anomalies, i.e. the inverse FIP effect, declines; however, clear deviations from solar abundances remain.
\end{abstract}

Key words. stars: abundances - stars: activity - stars: coronae - stars: late-type - stars: individual: EQ Pegasi - X-rays: stars

\section{Introduction}

M dwarfs populate the low-mass and low-temperature end of the main sequence, with masses of approximately $0.6 M_{\odot}$ and an effective temperature of about $3800 \mathrm{~K}$ at a spectral type of M0, down to $0.1 M_{\odot}$ and $2200 \mathrm{~K}$ at spectral type M9. Conspicuous changes in the optical spectra become apparent in the later subclasses as more and more molecules form with decreasing photospheric temperature, and molecular bands start to dominate. Dust formation marks the transition from late $\mathrm{M}$ dwarfs to the brown dwarf regime of $\mathrm{L}$ and $\mathrm{T}$ dwarfs.

Somewhat surprisingly, all $\mathrm{M}$ dwarfs seem to show at least some activity phenomena, and in particular active $\mathbf{M}$ dwarfs of spectral types M0-M4 are strong coronal X-ray sources with X-ray luminosities often close to a saturation limit of $\log L_{X} / L_{\text {bol }} \sim-3.3$ (Fleming et al. 1993; Pizzolato et al. 2003). Frequent and strong flaring with increases in X-ray luminosity by more than two orders of magnitude (e.g. Favata et al. 2000; Güdel et al. 2002a; Schmitt et al. 2008) is widespread among these stars, with additional variability on all timescales and amplitude levels. Their emission measure distributions peak around 6-8 MK (Robrade \& Schmitt 2005), thus their average coronal temperatures are much higher than solar (peak temperature 12 MK, see e.g. Brosius et al. 1996). Temperatures in excess of several $10 \mathrm{MK}$ are typically measured during larger flare events (Robrade \& Schmitt 2005).
On the other hand, late M dwarfs with spectral types M6-M9 are difficult to detect in X-rays at least during quiescence, yet their ability to produce transient X-ray luminosity enhancements by orders of magnitude during flares (Rutledge et al. 2000; Schmitt \& Liefke 2002; Stelzer et al. 2006) remains. Intermediate objects with a spectral type of $\approx$ M5 have lower coronal temperatures than the more active early $\mathrm{M}$ dwarfs, the permanent state of variability is replaced by periods of quiescence interrupted by individual flares, and the overall X-ray luminosity starts to decrease by more than an order of magnitude at around spectral type M5 (Güdel et al. 2002a; Fuhrmeister et al. 2007).

The abundance patterns observed in stellar coronae also appear to be related to changes in the coronal activity level. On the one hand, there is the so-called FIP effect observed in (comparably) inactive stars like the Sun (Feldman \& Laming 2000) or e.g. $\alpha$ Cen (Raassen et al. 2003), with the coronal abundances of elements with low first ionization potential (FIP) enhanced with respect to solar photospheric values. On the other hand, very active stars tend to show the inverse effect, i.e. elements with low FIP depleted and elements with a high FIP enhanced. Güdel et al. (2002b) and Telleschi et al. (2005) found that the solar-like FIP effect to first disappear and then reverse with increasing activity for solar analogs at different ages. This correlation can be expected to also apply to the sequence of M dwarfs: while the active early $M$ dwarfs are well-known to show the inverse FIP effect (see e.g. Robrade \& Schmitt 2005), 
Fuhrmeister et al. (2007) found the M5.5 star CN Leo to show much less pronounced abundance anomalies. Unfortunately, high-resolution X-ray spectra for intermediate objects are rare; and due to their low X-ray luminosity, it is in most cases impossible to obtain spectra suitable for a reasonable abundance analysis for late $\mathrm{M}$ dwarfs to confirm this trend.

Stellar coronal X-ray emission is associated with the production of magnetic fields driven by a dynamo mechanism (Parker 1955; Moffatt 1978), which is thought to be located in the transition layers between the rigidly rotating radiative core and the outer convection zone that rotates differentially for solarlike stars. However, models of the stellar interior predict that $\mathrm{M}$ dwarfs of spectral types later than $\approx \mathrm{M} 3$ become fully convective (Chabrier \& Baraffe 1997), leaving no shear layers between a radiative core and the convection zone behind. The solar-like $\alpha \omega$-dynamo thus does not work for these stars, and no dynamoinduced X-ray emission would be expected. Yet, the observed decline in X-ray luminosity around spectral type M5-M6 fits the finding of Mullan \& MacDonald (2001) that the influence of magnetic fields can shift the fully convective cut-off towards later spectral types. Fully convective stars produce less quiescent X-ray emission, but other activity indicators like $\mathrm{H} \alpha$ emission even increase in strength towards the low-mass L dwarfs. Alternative dynamo mechanisms, driving the required magnetic fields, have been proposed for these objects (e.g. Durney et al. 1993). In these scenarios with $\alpha^{2}$ or turbulent dynamos, the magnetic fields are sustained by small-scale velocity fields, while large-scale rotation plays only a minor role. As a consequence, no activity cycles and magnetic structures of small size and with a more uniform surface distribution are expected. However, most recent non-linear 3D simulations of dynamo action in fully convective stars (e.g. Chabrier \& Küker 2006; Dobler et al. 2006; Browning 2008) have shown that also large-scale fields could be produced in fast-rotating late $\mathrm{M}$ dwarfs, but these models disagree about whether or not the magnetic fields have axisymmetric components and/or a cyclic behavior.

With the required change of the dominating dynamo mechanism operating in early and late $\mathrm{M}$ dwarfs in mind, changes in the abundance patterns between these objects are of particular interest to characterize the coronae induced by the different dynamos. The above considerations concerning luminosity demand investigations of coronal properties of $\mathbf{M}$ dwarfs located close to the expected transition. The binary system EQ Peg consists of an M3.5 and M4.5 star and thus provides the rare opportunity to compare two such stars, which should otherwise be very similar since they are co-eval.

In the following, we present our Chandra HETGS observations of EQ Peg, that allows us to perform a separate spectral analysis of the two components. We also compare our observations of EQ Peg A and B to HETGS spectra of other M dwarfs covering a range of spectral types. We propose a classification of the coronal properties of active $\mathrm{M}$ dwarfs based on their spectral type.

\section{The EQ Peg binary system}

The visual binary system EQ Peg consists of two M dwarfs at a distance of $6.25 \mathrm{pc}$ with an angular separation of $\approx 6^{\prime \prime}$, i.e. EQ Peg A with spectral type M3.5 and an apparent magnitude $V_{\mathrm{A}}=10.35$ and EQ Peg B with spectral type M4.5 and magnitude $V_{\mathrm{B}}=12.4$. Both components are well-known (optical) flare stars (Pettersen 1976; Lacy et al. 1976). Jackson et al. (1989) observed microwave emission during quiescence, which they attributed to the brighter A component. Norton et al. (2007)
Table 1. Observation log for EQ Peg.

\begin{tabular}{ccc}
\hline \hline ObsID & Observation time & Exp. time [ks] \\
\hline 8453 & 2006-11-28T01:51-2006-11-28T22:48 & 29.95 \\
8484 & 2006-11-29T16:33-2006-11-29T08:59 & 10.95 \\
8485 & 2006-11-30T16:59-2006-12-01T08:54 & 30.94 \\
8486 & 2006-12-03T07:55-2006-12-03T09:02 & 27.95 \\
\hline
\end{tabular}

find a photometric period of 1.0664 days for the EQ Peg system from data of the SuperWASP transit survey, however, due to the large aperture size of SuperWASP, this period cannot be clearly assigned to one of the two components of EQ Peg, nor to two other stars in the field of view. However, Zboril \& Byrne (1998) find a rotational velocity of $14 \mathrm{~km} \mathrm{~s}^{-1}$ for EQ Peg A, while Delfosse et al. (1998) analyze only the B component and find $24.2 \pm 1.4 \mathrm{~km} \mathrm{~s}^{-1}$; both $v \sin i$ values are rather high and thus consistent with a short rotation period.

The EQ Peg system is a strong X-ray and EUV source with a number of flares recorded, but previous missions were not able to angularly resolve the two stars. Pallavicini et al. (1990) discuss two flares observed with EXOSAT. The first one, with an atypically shaped lightcurve, was observed by Haisch et al. (1987) in the context of a simultaneous EXOSAT and IUE campaign. A second large amplitude flare was observed by Pallavicini et al. (1986). Katsova et al. (2002) observed another large flare with ROSAT with simultaneous optical photometry. All of these flares were attributed to the A component. Monsignori Fossi et al. (1995) observed another set of flares on EQ Peg with EUVE and derived a differential emission measure distribution, based on line flux measurements, with a peak at about $10 \mathrm{MK}$, which is, however, not well constrained. A first approach to separate the two components in X-rays was undertaken with XMM-Newton (Robrade et al. 2004). Although the two stars show considerable overlap owing to the instrumental point spread function, it was possible to attribute about three quarters of the overall X-ray flux to the A component. A subsequent detailed spectral analysis without resolving the binary has been performed by Robrade \& Schmitt (2005). Our Chandra HETGS observations are the first $\mathrm{X}$-ray observations that allow an unambiguous spectral separation of the two binary components.

\section{Chandra HETGS observations of EQ Peg A and B}

\subsection{Observations and data analysis}

EQ Peg was observed on four occasions between 2006 November 28th and December 3rd with a total exposure time of $100 \mathrm{ks}$ with the Chandra High Energy Transmission Grating Spectrometer (HETGS); a journal of the observations is presented in Table 1. The HETGS consists of two grating arms, the Medium Energy Grating (MEG) and the High Energy Grating (HEG), which provide X-ray spectra in the wavelength range between $\approx 1.5$ and $30 \AA$ with medium (MEG: $0.023 \AA$ FWHM) and high (HEG: $0.012 \AA$ FWHM) resolution ${ }^{1}$. For data reduction we used the CIAO software version 3.4.

The separation of the 0th order images of EQ Peg A and B is 5.8" in the ACIS-S image, and therefore the dispersed spectra of the two stars can be easily separated with only a small overlap of the HEG and MEG grating arms at the shortest wavelengths $(<1.5 \AA)$. We extracted 1 st order HEG and MEG spectra

1 For a detailed description of the Chandra HETGS see the Chandra Proposers' Observatory Guide at http://asc.harvard. edu/proposer/POG/html/ 
EQ Peg ACIS-S pile-up corrected lightcurves

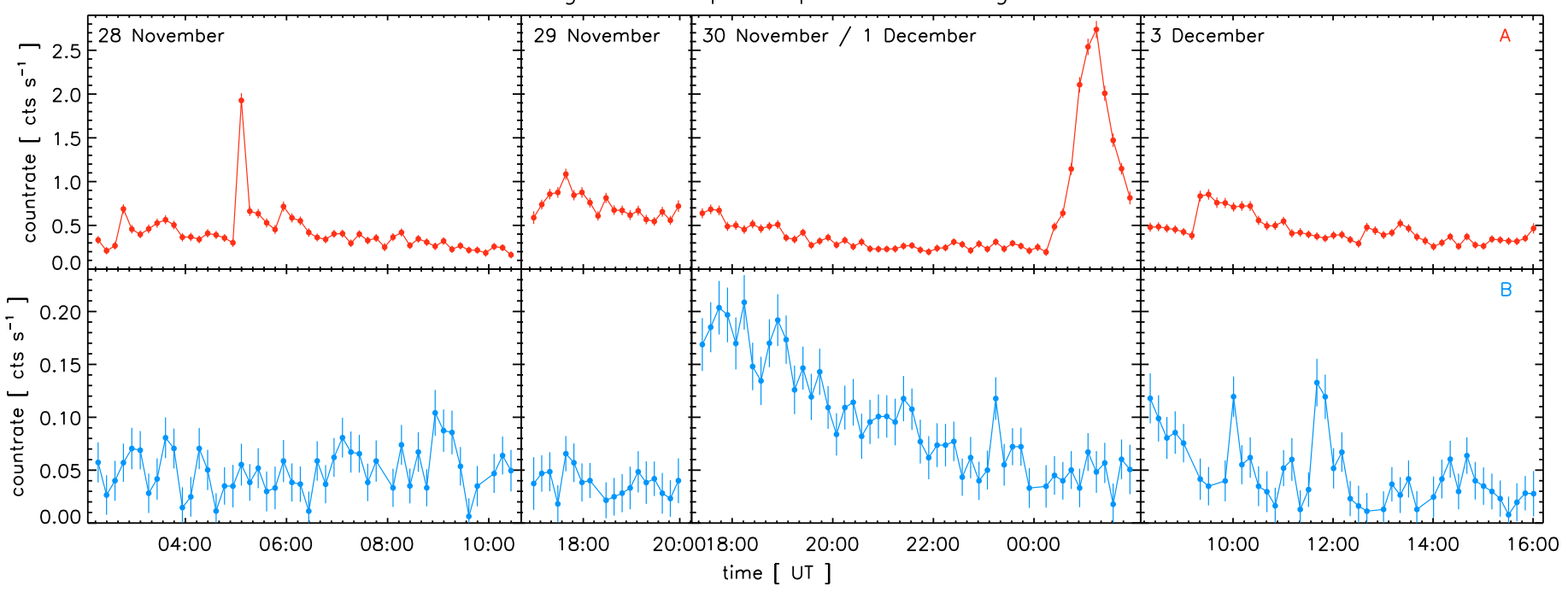

Fig. 1. Background-subtracted and pileup-corrected lightcurves for EQ Peg A (top) and B (bottom). Binsize for both sources is $600 \mathrm{~s}$.

for each component from each of the four datasets using the standard CIAO tools to obtain grating spectra for multiple sources.

For our final analysis we created separate HEG and MEG spectra for EQ Peg A and B, where we combined all four observations and the plus- and minus dispersion orders. Only for EQ Peg A have we also inspected the spectra from the individual observations.

For our final analysis, we created separate HEG and MEG spectra for EQ Peg A and B, where we combined all four observations and the plus and minus dispersion orders. Only for EQ Peg A we have also inspected the spectra from the individual observations. All line fluxes were measured with our CORA program (Ness \& Wichmann 2002), fitting the lines with Moffat line profiles given by

$I(\lambda)=I_{\max }\left(1+\left(\frac{\lambda-\lambda_{0}}{\Delta \lambda}\right)^{2}\right)^{-\beta}$

i.e., modified Lorentzians, with an exponent $\beta=2.4$ and a fixed line width of $0.02 \AA$ for MEG and $0.01 \AA$ for HEG spectra.

\subsection{Lightcurves}

We first extracted background-subtracted lightcurves for EQ Peg A and B from the dispersed and non-dispersed (0th order) photons. In order to obtain equivalent lightcurves of the two stars with maximized signal-to-noise, we use total (i.e. 0th order + dispersed) photons of EQ Peg B and the dispersed photons of EQ Peg A, scaled by the ratio of total and dispersed photons from EQ Peg B (assuming a similar spectral energy distribution for the two stars), as the the 0th order of EQ Peg A is strongly affected by pileup. The resulting lightcurves are shown in Fig. 1 for the four observation intervals.

EQ Peg A shows several flares. The most conspicuous event started at 0:00 UT on December 1st with a relatively slow rise and lasted about $2 \mathrm{~h}$. Unfortunately, the flare decay phase was not observed completely, since the observation ended before quiescence was reached again. At flare peak, the flux increases by a factor of six, and the flare is strong enough to allow the extraction of separate MEG spectra. Additional smaller flares occurred on November 28th at 5:00 UT, with a flux increase of about a factor of four, lasting about half an hour, and on
December 3rd at about 9:00 UT, with an increase by a factor of 2 , and a longer decay of more than $2 \mathrm{~h}$. The underlying quiescent level is difficult to determine owing to a large number of small-scale variations and apparently different quiescent emission levels during the four individual observations, with the base level for the A component at $\sim 0.3-0.4 \mathrm{cts} \mathrm{s}^{-1}$. Apart from pure statistical noise, such low-level amplitude variations can be caused either by small-scale flares or the evolution of active regions on the stellar surface. On November 29th the emission level was much higher than this quiescent level despite a lack of clear flare-like signatures, which might be explained by active regions newly emerged or rotated into the field of view, while on November 30th a slow decrease in count rate is observed that might be attributed to a long-duration flare. The B component at $\sim 0.05 \mathrm{cts} \mathrm{s}^{-1}$ is much fainter and does not show conspicuous short duration flares, except on Dec 3rd. On November 30th the count rate of EQ Peg B slowly decreased during the whole 8 hours of the observation and again we may have witnessed the decay of a flare.

We computed median and mean count rates in order to compare the flux levels of the two components outside obvious flaring periods, and find the quiescence level of EQ Peg B lower than that of $\mathrm{A}$ by factors of about 6 to 10. Both stars show a decay in count rate on November 30th, however, a direct connection of flare activity on the two components is difficult to envisage considering the distance of $\sim 36$ AU between $\mathrm{A}$ and $\mathrm{B}$, although a similar correlated behavior of the system with simultaneous quiescent and flaring states has been observed by Robrade et al. (2004) with XMM-Newton. These authors also found a lower flux ratio of 4-5 during quiescence and 2.5-3 during flaring states. However, due to the limited spatial resolution of XMM's EPIC detectors, the exact brightness ratios are less certain.

We also extracted the lightcurves in different energy bands to obtain time-resolved hardness ratios. These give us the temperature evolution of the plasma. While there is no unique temperature that describes the spectrum (see Sect. 4.2), the temperature distribution cannot be mapped as a function of time, and the differential emission measure distribution can only by determined as an average over the entire observation. Because of pileup in the 0th order of the A component, we extracted the 


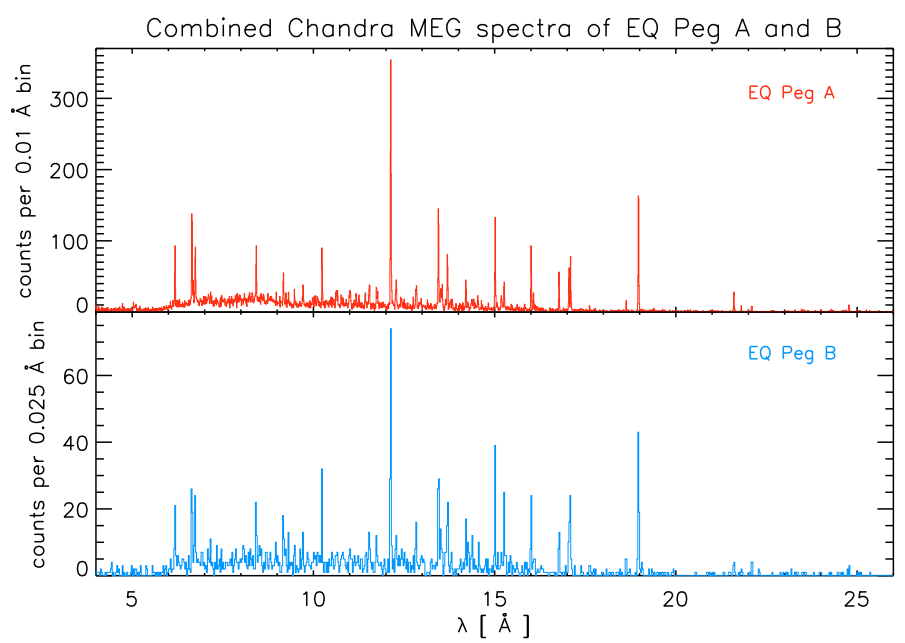

Fig. 2. Chandra MEG spectra of EQ Peg A (top, red) and B (bottom, blue). Both spectra have been rebinned.

hardness ratios only from the dispersed photons for both stars. With the hard and soft bands chosen to range from 1.0-10.0 keV and $0.3-1.0 \mathrm{keV}$ respectively, the two components show consistent hardness ratios during quiescence, with values ranging from 0.25 to 0.5 , indicating a similar spectral energy distribution. Averaged over the total observing time, the hardness ratio for both stars is 0.43 ; a spectral hardening to values up to 0.8 is apparent during the major flares on the A component. For our spectral analysis, we considered only the total spectrum of EQ Peg B, while for EQ Peg A we considered the total spectrum and a "quiescent" spectrum, where we excluded the two larger flares on November 28th and December 1st.

We computed overall X-ray luminosities $\log L_{X}$ of 28.71 for EQ Peg A (28.67 for the quiescent state only) and 27.89 for EQ Peg B from the integrated dispersed photons in the MEG spectra over a wavelength range from 2 to $25 \AA$, i.e. in this spectral range, the A component is by a factor of 6-7 more luminous than the B component. However, following Kenyon \& Hartmann (1995), the bolometric luminosities of the two stars are $\log L_{\text {bol }} \approx 31.90$ and 31.28 . This results in $\log L_{\mathrm{X}} / L_{\text {bol }}$ values of -3.19 ( -3.23 during quiescence) and -3.39 for EQ Peg A and $\mathrm{B}$ respectively, i.e. both stars are located in the saturation regime of X-ray emission (Pizzolato et al. 2003).

\subsection{Grating spectra}

In Fig. 2 we show the Chandra MEG spectra of EQ Peg A (top) and B (bottom). Both stars show well-pronounced continua between $5 \AA$ and $15 \AA$, indicating larger amounts of hightemperature plasma in their coronae. The strongest lines in the total MEG count spectra are $\mathrm{Ne} \mathrm{X}$ and $\mathrm{O}$ VIII Ly $\alpha$, other prominent lines originate from Si XIV and XIII, Ne IX, and Fe XVII. The iron lines tend to be somewhat stronger in EQ Peg B. The O VII triplet lines are clearly detected but suffer from the low effective area of the MEG at long wavelengths. The weak O VII $1 \mathrm{~s}-3$ p line at $18.63 \AA$ is also detected in both stars. After conversion of counts to photon fluxes, the O VIII Ly $\alpha$ line is the strongest line in the spectra, and also the O VII flux is quite high, indicating the presence of considerable amounts of lowtemperature plasma.

In order to probe the shape of the continua and to directly compare the two binary components, we computed the cumulative count spectra from $4 \AA$ to $26 \AA$ from the combined count

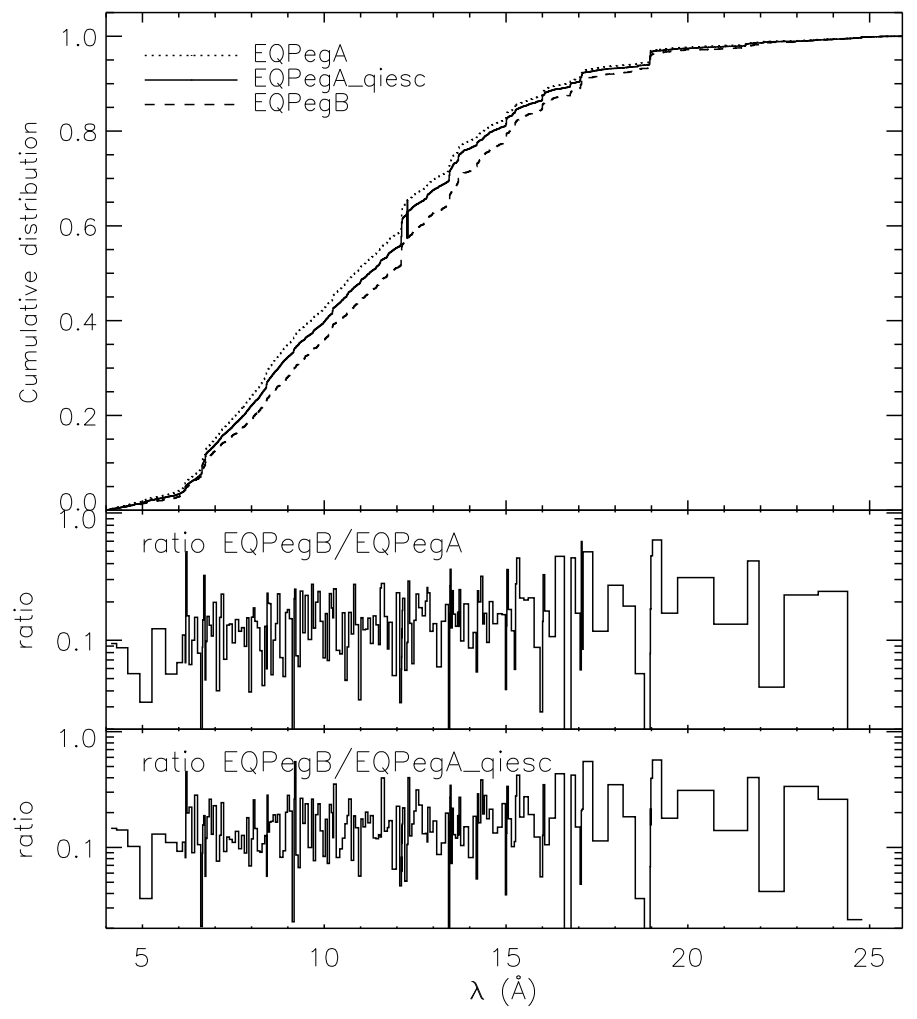

Fig. 3. Cumulative distribution (top) of counts in the MEG spectra of EQ Peg A (dotted line: all data, solid line: with the two larger flares on 28th November and December 1st excluded) and B (dashed line); and ratio B/A (middle panel: all data, bottom: with the two larger flares on EQ Peg A excluded).

spectra of EQ Peg A and B (top panel of Fig. 3). A steep rise of the cumulative spectrum indicates a harder spectrum and thus higher temperatures, as observed for EQ Peg A compared to EQ Peg B. Since this may reflect the strong flares observed on the A component, we considered also the quiescent spectrum for EQ Peg A. As can be seen from Fig. 3 (top), this spectrum is indeed closer to the B component, however, EQ Peg A still appears somewhat harder. When comparing the total spectra of $\mathrm{A}$ and $\mathrm{B}$, these differences are relatively small, and a KS test returns probabilities of around 3\% that the two stars are different in their spectral shape. We note that the presence of strong emission lines, e.g., Ne X at $12.12 \AA$, leads to steps in the cumulative spectrum. However, the depths of these steps seem similar for EQ Peg A and B, and the major differences in the cumulative distribution can be attributed to the continuum.

In Tables 2 and 3 we provide lists of the measured line counts and resulting photon fluxes for the combined spectra of EQ Peg A and B, and for the quiescent state of EQ Peg A, which we used in our subsequent analysis; note that a sophisticated procedure has been applied to deconvolve the blending of the Ne IX triplet and its contamination with iron lines, mainly from Fe XVII and Fe XIX (Ness et al. 2003b). In addition to the line fluxes of the three triplet constituents, we fitted global scaling factors to the line fluxes of the contamining ions observed by Ness et al. (2003b) in the well-exposed HETGS spectrum of Capella where the contamination is very strong. Additionally, the NeX line at $12.13 \AA$ has been corrected to account for a blend by Fe XVII at $12.12 \AA$. According to CHIANTI 5.2 (Landi et al. 2006), using the ionization balance from Mazzotta et al. (1998), the line emissivity of the contaminating line is 
Table 2. Measured line counts and photon fluxes (in $10^{-5}$ photons $\mathrm{cm}^{-2} \mathrm{~s}^{-1}$ ) in the combined Chandra MEG spectra. Flux predictions from the reconstructed DEMs (see Sect. 4.2) are also listed.

\begin{tabular}{|c|c|c|c|c|c|c|c|c|c|}
\hline \multirow{2}{*}{$\begin{array}{r}\lambda \lambda \\
{[\AA]}\end{array}$} & \multirow[t]{2}{*}{ Ion } & \multicolumn{4}{|c|}{ 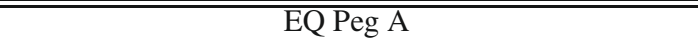 } & \multicolumn{4}{|c|}{$\overline{\text { EQ Peg B }}$} \\
\hline & & Counts & Photon fluxes & Method 1 & Method 2 & Counts & Photon fluxes & Method 1 & Method 2 \\
\hline 4.73 & S XVI & $22.3 \pm 5.6$ & $0.61 \pm 0.15$ & 0.42 & 0.32 & - & - & $\overline{-}$ & $\overline{-}$ \\
\hline 5.04 & $\mathrm{SxV}$ & $28.7 \pm 6.3$ & $0.84 \pm 0.18$ & 0.92 & 0.94 & - & - & - & - \\
\hline 6.18 & Si XIV & $176.6 \pm 14.6$ & $1.78 \pm 0.15$ & 1.92 & 1.86 & $30.4 \pm 5.9$ & $0.31 \pm 0.06$ & 0.21 & 0.13 \\
\hline 6.65 & Si XIII & $321.6 \pm 19.7$ & $2.74 \pm 0.17$ & 2.71 & 2.75 & $40.4 \pm 6.8$ & $0.34 \pm 0.06$ & 0.36 & 0.37 \\
\hline 6.69 & Si XIII & $61.7 \pm 10.6$ & $0.53 \pm 0.09$ & 0.72 & 0.73 & $5.0 \pm 3.4$ & $0.04 \pm 0.03$ & 0.10 & 0.10 \\
\hline 6.74 & Si XIII & $202.5 \pm 16.2$ & $1.58 \pm 0.13$ & 1.31 & 1.33 & $25.7 \pm 6.0$ & $0.20 \pm 0.05$ & 0.19 & 0.19 \\
\hline 8.42 & Mg XII & $178.9 \pm 15.9$ & $1.45 \pm 0.13$ & 1.58 & 1.60 & $30.2 \pm 6.0$ & $0.24 \pm 0.05$ & 0.25 & 0.23 \\
\hline 9.17 & Mg XI & $107.7 \pm 12.4$ & $1.58 \pm 0.18$ & 1.54 & 1.50 & $26.0 \pm 5.6$ & $0.38 \pm 0.08$ & 0.32 & 0.34 \\
\hline 9.23 & Mg XI & $33.9 \pm 8.2$ & $0.51 \pm 0.12$ & 0.36 & 0.35 & $6.7 \pm 3.4$ & $0.10 \pm 0.05$ & 0.08 & 0.08 \\
\hline 9.31 & Mg XI & $34.6 \pm 8.4$ & $0.55 \pm 0.13$ & 0.81 & 0.78 & $11.2 \pm 3.9$ & $0.18 \pm 0.07$ & 0.17 & 0.18 \\
\hline 10.24 & $\mathrm{Ne} X$ & $160.8 \pm 14.4$ & $2.42 \pm 0.22$ & 3.05 & 3.07 & $29.4 \pm 5.91$ & $0.44 \pm 0.09$ & 0.34 & 0.36 \\
\hline 11.17 & Fe XXIV & $47.2 \pm 9.7$ & $0.95 \pm 0.19$ & 0.48 & 0.44 & - & - & 0.02 & 0.01 \\
\hline 11.55 & Ne IX & $101.3 \pm 12.3$ & $2.13 \pm 0.26$ & 1.80 & 1.84 & $19.5 \pm 5.12$ & $0.41 \pm 0.11$ & 0.28 & 0.28 \\
\hline 11.74 & Fe XXIII & $65.1 \pm 10.5$ & $1.50 \pm 0.24$ & 0.91 & 1.00 & - & - & 0.13 & 0.09 \\
\hline 11.78 & Fe XXII & $44.9 \pm 9.6$ & $1.11 \pm 0.24$ & 1.12 & 1.29 & - & - & 0.11 & 0.17 \\
\hline 12.13 & $\mathrm{NeX}$ & $891.8 \pm 29.9$ & $24.47 \pm 0.82$ & 24.00 & 24.06 & $104.8 \pm 10.6$ & $2.88 \pm 0.29$ & 2.75 & 2.92 \\
\hline 13.45 & $\mathrm{Ne} I X$ & $320.2 \pm 17.9$ & $15.89 \pm 0.88$ & 17.02 & 16.58 & $51.4 \pm 7.5$ & $2.26 \pm 0.33$ & 2.66 & 2.56 \\
\hline 13.55 & $\mathrm{Ne} I X$ & $77.2 \pm 8.8$ & $3.28 \pm 0.37$ & 3.67 & 3.57 & $9.1 \pm 3.0$ & $0.39 \pm 0.13$ & 0.56 & 0.56 \\
\hline 13.69 & $\mathrm{Ne} I X$ & $184.0 \pm 13.6$ & $8.90 \pm 0.66$ & 9.53 & 9.28 & $32.0 \pm 5.7$ & $1.55 \pm 0.28$ & 1.45 & 1.46 \\
\hline 14.21 & Fe XVIII & $92.2 \pm 11.1$ & $5.80 \pm 0.70$ & 4.77 & 4.86 & $18.7 \pm 4.6$ & $1.18 \pm 0.29$ & 0.70 & 0.76 \\
\hline 15.01 & Fe XVII & $270.1 \pm 17.3$ & $15.72 \pm 1.01$ & 18.74 & 18.51 & $43.2 \pm 7.0$ & $2.51 \pm 0.40$ & 3.29 & 3.31 \\
\hline 15.26 & Fe XVII & $102.2 \pm 9.3$ & $6.60 \pm 0.60$ & 5.36 & 5.29 & $26.5 \pm 5.7$ & $1.71 \pm 0.36$ & 0.95 & 0.95 \\
\hline 16.01 & O VIII & $193.1 \pm 14.5$ & $13.71 \pm 1.03$ & 8.40 & 8.33 & $27.2 \pm 5.7$ & $1.93 \pm 0.40$ & 0.89 & 1.03 \\
\hline 16.78 & Fe XVII & $116.4 \pm 11.3$ & $10.36 \pm 1.00$ & 11.41 & 11.25 & $18.3 \pm 4.6$ & $1.63 \pm 0.41$ & 2.05 & 2.04 \\
\hline 17.05 & Fe XVII & $144.7 \pm 12.7$ & $14.36 \pm 1.26$ & 14.51 & 14.30 & $24.8 \pm 5.2$ & $2.46 \pm 0.52$ & 2.60 & 2.59 \\
\hline 17.10 & Fe XVII & $139.3 \pm 12.4$ & $14.07 \pm 1.25$ & 12.10 & 11.93 & $26.2 \pm 5.4$ & $2.65 \pm 0.55$ & 2.15 & 2.16 \\
\hline 18.63 & O VII & $33.9 \pm 6.2$ & $4.78 \pm 0.87$ & 3.37 & 3.43 & $7.3 \pm 3.1$ & $1.03 \pm 0.43$ & 0.51 & 0.48 \\
\hline 18.97 & O VIII & $435.8 \pm 21.1$ & $71.90 \pm 3.48$ & 70.50 & 69.90 & $63.4 \pm 8.2$ & $10.46 \pm 1.36$ & 7.77 & 8.82 \\
\hline 21.60 & O VII & $69.6 \pm 8.6$ & $24.21 \pm 2.98$ & 30.53 & 31.30 & $7.6 \pm 2.8$ & $1.56 \pm 0.91$ & 4.78 & 4.40 \\
\hline 21.81 & O VII & $14.2 \pm 4.0$ & $5.64 \pm 1.59$ & 6.83 & 7.07 & $3.7 \pm 2.0$ & $1.47 \pm 0.79$ & 1.45 & 0.99 \\
\hline 22.10 & O VII & $24.0 \pm 5.1$ & $10.95 \pm 2.33$ & 18.08 & 18.70 & $10.3 \pm 3.5$ & $4.70 \pm 1.6$ & 3.71 & 2.62 \\
\hline 24.79 & N VII & $19.3 \pm 4.5$ & $7.84 \pm 1.85$ & 7.84 & 7.84 & $2.0 \pm 1.7$ & $0.80 \pm 0.72$ & 0.80 & 0.80 \\
\hline
\end{tabular}

comparable to the line at $12.26 \AA$, and the ratio of these two lines shows no dependence on electron density and only a mild dependence on electron temperature. Additionally, both transitions go to the ground state and share the same electron configuration in their excited states. We thus subtracted the line counts measured in the line at $12.26 \AA$ from the Ne $\mathrm{X}$ line as a proxy for the unresolved blending line at $12.12 \AA$. Figure 4 illustrates the fit for the total HEG and MEG spectra of EQ Peg A.

\section{Results}

\subsection{Coronal densities}

Using the forbidden and intercombination lines of the heliumlike triplets of silicon, magnesium, neon, and oxygen, we computed the density-sensitive $f / i$-ratios of these ions for EQ Peg A and B. In Table 4 we list these ratios with the respective peak formation temperatures of the ion and the derived electron densities $n_{\mathrm{e}}$ for EQ Peg A and B. For the conversion of the measured $f / i$ ratios to densities, we used the relation

$\frac{f}{i}=\frac{R_{0}}{1+n_{\mathrm{e}} / N_{\mathrm{c}}}$

with the low-density limit $R_{0}$ and the critical density $N_{\mathrm{c}}$ where we adopted the values from Table 5 in Ness et al. (2002). Theoretical modeling of the $f / i$ ratios still suffers from the incompleteness of current atomic databases, as contributions from satellite lines and effects from dielectronic recombination have to be taken into account.

The uncertainty in the density derived from O VII is large, as a result of the low effective area at long wavelengths. While the Ne IX triplet around $13.5 \AA$ is well exposed, severe contamination complicates the line flux measurements, especially of the Ne IX i line at $13.54 \AA$ (Ness et al. 2003b), see also Sect. 3.3. The density value derived for EQ Peg A is higher than the one derived from O VII. Since Ne IX traces higher coronal temperatures, this may indicate a higher pressure in the hotter plasma regions, however, the uncertainties do not include uncertainties from the deblending procedure. The $f / i$-ratio derived for the $\mathrm{B}$ component is - nominally - in the low-density limit region, however, the error is so large that the same density derived for the A component is also consistent with the data. The Mg and Si triplets are much stronger affected by satellite lines. Additionally, the Mg XI triplet is blended with higher-order lines from the Lyman series of $\mathrm{Ne} \mathrm{X}$ as discussed by Testa et al. (2004). The flux in the magnesium triplet lines is too low in both components of the EQ Peg binary for a deblending procedure to yield any useful refinements. Since neon turns out to be far more abundant than magnesium (see Sect. 4.2), the blending contributions from neon amounts to a significant fraction of the line fluxes, and we consider the high densities derived from magnesium not reliable. Consistent with that interpretation, the Si XIII triplet lines are consistent with being in the low-density limit, which is not surprising since the density-sensitive range for this ion is higher than the densities normally encountered in stellar coronae. However, the Si triplet 
Table 3. Measured line counts and photon fluxes in the Chandra MEG spectra of the quiescent state of EQ Peg A.

\begin{tabular}{rrrr}
\hline \hline$\lambda[\AA]$ & Ion & Counts & Fluxes \\
\hline 4.73 & S XVI & $8.6 \pm 4.1$ & $0.25 \pm 0.12$ \\
5.04 & S XV & $22.6 \pm 5.8$ & $0.70 \pm 0.18$ \\
6.18 & Si XIV & $143.7 \pm 13.2$ & $1.54 \pm 0.14$ \\
6.65 & Si XIII & $317.7 \pm 19.1$ & $2.87 \pm 0.17$ \\
6.69 & Si XIII & $57.0 \pm 10.1$ & $0.52 \pm 0.09$ \\
6.74 & Si XIII & $193.6 \pm 15.6$ & $1.60 \pm 0.13$ \\
8.42 & Mg XII & $175.4 \pm 15.3$ & $1.51 \pm 0.13$ \\
9.17 & Mg XI & $98.7 \pm 11.8$ & $1.54 \pm 0.18$ \\
9.23 & Mg XI & $27.5 \pm 8.1$ & $0.44 \pm 0.13$ \\
9.31 & Mg XI & $31.9 \pm 8.4$ & $0.54 \pm 0.14$ \\
10.24 & NeX & $155.6 \pm 14.0$ & $2.49 \pm 0.22$ \\
11.17 & Fe XXIV & $20.6 \pm 7.6$ & $0.44 \pm 0.16$ \\
11.55 & Ne IX & $90.3 \pm 11.5$ & $2.02 \pm 0.26$ \\
11.74 & Fe XXIII & $45.9 \pm 9.0$ & $1.12 \pm 0.22$ \\
11.78 & Fe XXII & $42.4 \pm 9.0$ & $1.11 \pm 0.24$ \\
12.13 & Ne X & $813.8 \pm 28.5$ & $23.75 \pm 0.83$ \\
13.45 & Ne IX & $293.4 \pm 17.1$ & $13.67 \pm 0.80$ \\
13.55 & Ne IX & $61.1 \pm 7.8$ & $2.76 \pm 0.35$ \\
13.69 & Ne IX & $169.1 \pm 13.0$ & $8.69 \pm 0.67$ \\
14.21 & Fe XVIII & $82.7 \pm 10.6$ & $5.53 \pm 0.71$ \\
15.01 & Fe XVII & $266.3 \pm 16.9$ & $16.45 \pm 1.05$ \\
15.26 & Fe XVII & $92.8 \pm 10.7$ & $7.39 \pm 0.77$ \\
16.01 & O VIII & $184.8 \pm 14.0$ & $13.69 \pm 1.05$ \\
16.78 & Fe XVII & $114.3 \pm 11.0$ & $10.80 \pm 1.04$ \\
17.05 & Fe XVII & $139.9 \pm 12.6$ & $14.73 \pm 1.33$ \\
17.10 & Fe XVII & $137.2 \pm 12.4$ & $14.70 \pm 1.33$ \\
18.63 & O VII & $30.3 \pm 5.6$ & $4.55 \pm 0.87$ \\
18.97 & O VIII & $414.0 \pm 20.5$ & $72.49 \pm 3.60$ \\
21.60 & O VII & $64.5 \pm 8.3$ & $23.76 \pm 3.06$ \\
21.81 & O VII & $14.0 \pm 4.0$ & $5.91 \pm 1.69$ \\
22.10 & O VII & $24.0 \pm 5.1$ & $11.62 \pm 2.47$ \\
24.79 & N VII & $15.0 \pm 4.3$ & $6.47 \pm 1.85$ \\
\hline & & &
\end{tabular}

EQ Peg A: Ne X and Fe XVII 12.26/Fe XXI 12.28

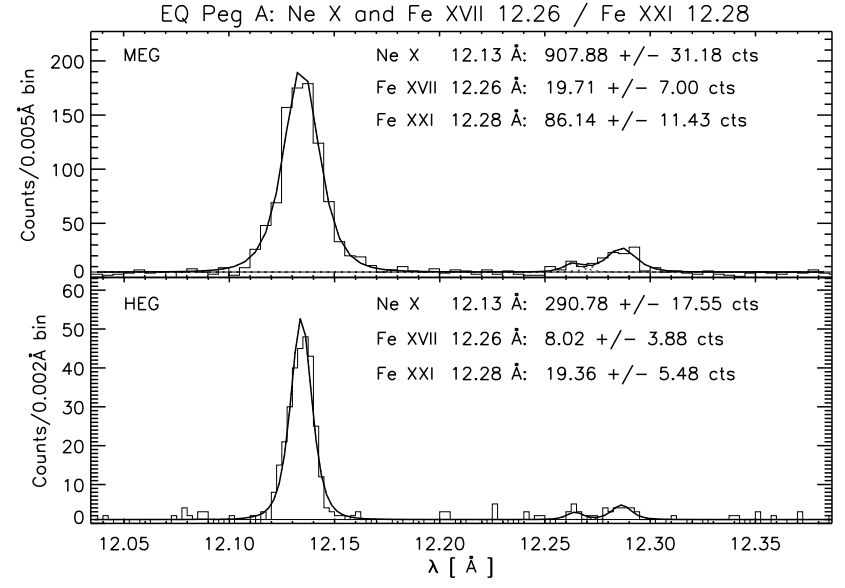

Fig. 4. Spectral region around the NeX line at $12.13 \AA$ and the Fe XVII and Fe XXI lines at 12.26 and 12.28 $\AA$ in the total spectrum of EQ Peg A.

is also problematic because of a steep increase of the effective area in both the HEG and MEG that occurs exactly at the wavelength of the forbidden line.

The flux in the Ne IX triplet of EQ Peg A is large enough to investigate not only the density in the combined spectrum, but also in the individual datasets. In Fig. 5 we show the corresponding spectral region for each of our four data sets. While differences in the strength of the intercombination line at $13.55 \AA$ are
Table 4. $f / i$ ratios (in photon units) and coronal densities for EQ Peg A and B from total Chandra MEG spectra.

\begin{tabular}{rrrrrr}
\hline \hline & & \multicolumn{2}{c}{ EQ Peg A } & \multicolumn{2}{c}{ EQ Peg B } \\
ion & $\log T$ & $f / i$ & $\log n_{\mathrm{e}}$ & $f / i$ & $\log n_{\mathrm{e}}$ \\
\hline O VII & 6.3 & $1.69 \pm 0.60$ & $10.62_{-0.27}^{+0.29}$ & $2.81 \pm 0.87$ & $<10.98$ \\
Ne IX & 6.6 & $2.38 \pm 0.32$ & $11.40_{-0.22}^{+0.18}$ & $3.53 \pm 1.33$ & $<11.51$ \\
Mg XI & 6.8 & $1.01 \pm 0.35$ & $12.99_{-0.23}^{+0.27}$ & $1.68 \pm 1.04$ & $<13.28$ \\
Si XIII & 6.95 & $3.28 \pm 0.62$ & $<11.17$ & $5.08 \pm 3.64$ & $<13.52$ \\
\hline
\end{tabular}

Table 5. Absolute coronal abundances (relative to Asplund et al. 2005) of EQ Peg A and B for the total observation time, determined with the DEM methods.

\begin{tabular}{rrrrr}
\hline \hline & \multicolumn{2}{c}{ EQ Peg A } & \multicolumn{2}{c}{ EQ Peg B } \\
Element & Method 1 & Method 2 & Method 1 & Method 2 \\
\hline $\mathrm{N}$ & $0.42 \pm 0.10$ & $0.39 \pm 0.09$ & $0.16 \pm 0.15$ & $0.20 \pm 0.18$ \\
$\mathrm{O}$ & $0.42 \pm 0.04$ & $0.40 \pm 0.04$ & $0.15 \pm 0.09$ & $0.26 \pm 0.08$ \\
$\mathrm{Ne}$ & $1.14 \pm 0.07$ & $1.07 \pm 0.07$ & $0.93 \pm 0.07$ & $0.83 \pm 0.07$ \\
$\mathrm{Mg}$ & $0.22 \pm 0.01$ & $0.20 \pm 0.02$ & $0.28 \pm 0.04$ & $0.27 \pm 0.04$ \\
$\mathrm{Si}$ & $0.51 \pm 0.02$ & $0.46 \pm 0.02$ & $0.54 \pm 0.07$ & $0.52 \pm 0.07$ \\
$\mathrm{~S}$ & $0.64 \pm 0.16$ & $0.58 \pm 0.21$ & - & - \\
$\mathrm{Fe}$ & $0.19 \pm 0.02$ & $0.18 \pm 0.01$ & $0.20 \pm 0.02$ & $0.17 \pm 0.02$ \\
\hline
\end{tabular}

apparent in the individual datasets, no changes in density can be established at a statistically significant level. In addition to the complete dataset 8485 , we show also the spectrum extracted for the large flare (shaded in gray in the bottom left panel of Fig. 5). Dividing this observation in flare and non-flaring states gives $f / i$-ratios of $1.13 \pm 0.59$ and $2.24 \pm 0.61$, or density values $\log n_{\mathrm{e}}$ of $12.07_{-0.31}^{+0.43}$ and $11.49_{-0.43}^{+0.32}$, respectively. For the combined quiescent spectrum, we find $f / i=2.77 \pm 0.42$, corresponding to $\log n_{\mathrm{e}}=11.13_{-0.09}^{+0.29}$, which is lower but still consistent with the total spectrum (see Table 4) and clearly lower than during the large flare. This indicates activity-related density variations as observed by Maggio \& Ness (2005) on the active M dwarf AD Leo. Unambiguous measurements of coronal density variations have so far only been observed during very large flares on the active $\mathrm{M}$ dwarfs Proxima Centauri and CN Leo (Güdel et al. 2002a; Liefke et al. 2007). The two density values derived here for flare and quiescence are consistent with each other at the $2 \sigma$ level, however, the density values observed during flares on active stars in general - although consistent with the quiescent state - tend to result in higher densities (see e.g. Mitra-Kraev \& Ness 2006).

We also note the different levels of contamination of the neon triplet by Fe XVII (13.8 $\AA$ ) and Fe XIX (13.51 $\AA$ ) in the different datasets. During the large flare, this can be attributed on the one hand to the higher coronal temperatures increasing the amount of Fe XIX compared to other ionization stages of iron, and on the other hand possibly also to an increased $\mathrm{Fe} / \mathrm{Ne}$ abundance ratio during the flare, as discussed below.

\subsection{Differential emission measure}

In order to derive absolute abundances we investigated the differential emission measure (DEM) of the two stars from the line fluxes of the strongest density-insensitive lines listed in Table 2. For EQ Peg A, the HEG spectrum can also be used in addition to the MEG spectrum, while for EQ Peg B only the MEG spectrum provides data with a reasonable signal-to-noise ratio. In a two-step procedure we first reconstructed the DEM utilizing an abundance-independent approach similar to the one proposed by 


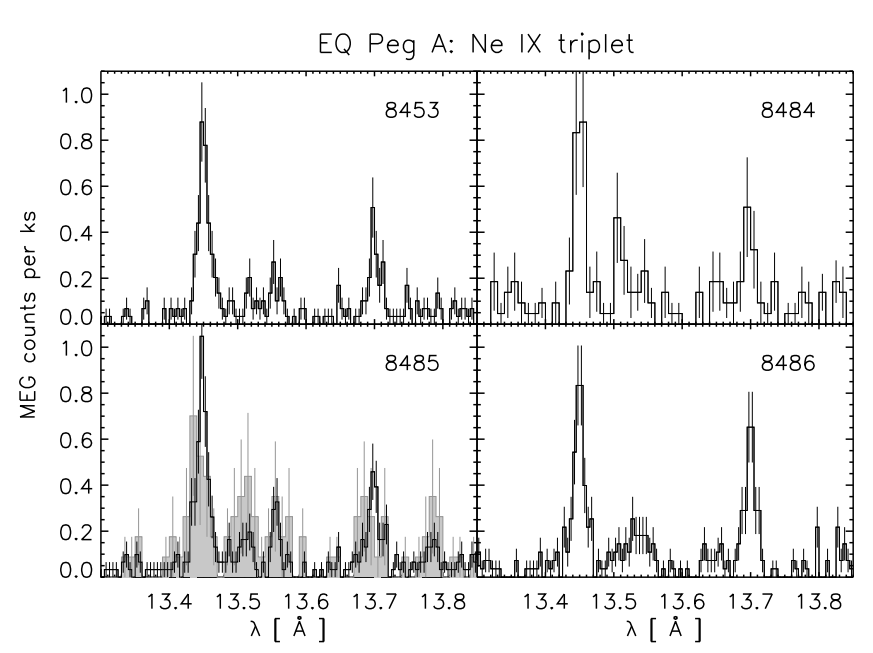

Fig. 5. The Ne IX triplet in the MEG spectra of EQ Peg A during the four observations. On observation No. 8485 the flare spectrum only is overlaid in gray.

Schmitt \& Ness (2004). In a second step we then determined the abundances from the ratios of measured line fluxes to those predicted by the DEM.

We used a volume differential emission measure defined as

$\operatorname{DEM}(T)=n_{\mathrm{e}}^{2} \frac{\mathrm{d} V}{\mathrm{~d} T}$

in units $\mathrm{cm}^{-3} \mathrm{~K}^{-1}$. The DEM is related to the theoretical photon flux $f_{j i}$ (in photons $\mathrm{cm}^{-2} \mathrm{~s}^{-1}$ ) of a spectral line originating from an atomic transition from the upper level $j$ to the lower level $i$ in the ion $X^{Z+}$ by

$f_{j i}=\frac{A_{X}}{4 \pi d^{2}} \int G(T) \operatorname{DEM}(T) \mathrm{d} T$

where $A_{X}$ is the abundance of element $X$ relative to solar photospheric values and the line contribution function $G(T)$ with

$G(T)=A_{j i} \frac{n_{j}\left(X^{Z+}\right)}{n\left(X^{Z+}\right)} \frac{n\left(X^{Z+}\right)}{n(X)} \frac{n(X)}{n(\mathrm{H})} \odot \frac{n(\mathrm{H})}{n_{\mathrm{e}}} \frac{1}{n_{\mathrm{e}}}$

$A_{j i}$ being the Einstein coefficient for spontaneous emission of the transition, $n_{j}\left(X^{Z+}\right) / n\left(X^{Z+}\right)$ the level population of the upper level, $n\left(X^{Z+}\right) / n(X)$ the ionization balance, $n(X) / n(\mathrm{H})_{\odot}$ the solar photospheric abundance, and $n(\mathrm{H}) / n_{\mathrm{e}}$ the proton-to-electron ratio, which is $\approx 0.83$ for coronal plasmas where hydrogen and helium are usually fully ionized. While the DEM describes the properties of the emitting plasma, the line contribution function summarizes constants and the underlying atomic physics. We used CHIANTI 5.2 (Landi et al. 2006) and the ionization balance of Mazzotta et al. (1998) to calculate $G(T)$ for each line.

Our abundance-independent reconstruction of the DEM is based on line ratios, and we used the ratios of the H-like Ly $\alpha$ and the He-like resonance lines of oxygen, neon, magnesium, and silicon, plus several ratios of adjacent ionization stages of iron from FeXVII to Fe XXIV. Since these line ratios involve only lines of the same element, the elemental abundances cancel out, and each ratio poses a constraint on the shape of the DEM. The choice of these lines yields a temperature coverage from $\approx 2$ to $20 \mathrm{MK}$, however, the low effective area at longer wavelengths with low-temperature lines like O VII and $\mathrm{O}$ VIII results in relatively large uncertainties at temperatures below 5 MK. For EQ Peg A, a total of 13 ratios have been used, while some of the ratios consisting of higher ionization stages of iron could not be used for the B component because of low signal, leaving only 9 usable ratios. In order to fix the absolute level of the DEM in addition to constraining the shape by the ratios, but still independently of a reference element like iron, we force the DEM and the theoretical continuum emissivity as implemented in CHIANTI to reproduce the observed continuum flux. For both components of EQ Peg, we used the wavelength region around $7.5 \AA$, where the effective area of the MEG reaches its maximum, and the spectrum is, according to the CHIANTI database, essentially line-free, exept for a few weak aluminum and magnesium lines. We assume that any remaining emission at these wavelengths originates from the continuum and neglect the contribution of any further weak transitions. As a result of merging the four datasets, the determined DEMs constitute an average over these observations with the different states of activity and quiescent and flaring periods of the two stars.

We used two slightly different approaches to model the DEM, that we parameterize by polynomials of different orders. In our first method (method 1) we fitted the polynomial parameters as $\log$ DEM, and with our second method (method 2) we model the linear DEM, both as a function of $\log T$. While for method 1 no boundary conditions are needed, for method 2 we forced the value of DEM to be zero at two variable temperatures, and positive between these temperatures. For a more detailed description we refer to Liefke \& Schmitt (in preparation).

For EQ Peg A, both methods give acceptable best-fits to the selected line ratios assuming 3rd and 5th order polynomials, yielding values of reduced $\chi^{2}$ of 2.06 and 2.36 for methods 1 and 2, respectively. These DEMs are shown in the top panel of Fig. 6. The polynomials are an analytical representation of the shape of the DEM, which is only valid for certain temperature range which is at first restricted by the temperature range covered by the emissivities of the lines implemented in the fit. In Fig. 6 we additionally plot the polynomials with solid lines in the temperature range we consider as well-constrained from the available lines, and with dashed or dotted lines outside this range. Since the high-temperature regime is better represented by the available ratios than the low-temperature range (see above), the two methods yield best agreement at high temperatures. However, the peaks in the DEM disagree from the two methods, and the poor constraints from temperatures $\log T$ below 6.5 lines make it difficult to quantify the exact amount of cool material; this may even falsely hint at a DEM peak at a lower temperature.

For EQ Peg B, this becomes even more apparent, see the bottom panel of Fig. 6 . The best-fit results are obtained with 4th order polynomials with both methods, with reduced $\chi^{2}$ values of 1.67 and 1.85; however, the two approaches give consistent results only in the range of $6.8<\log T<7.2$. The DEM reconstructed from method 2 shows an unphysical increase towards lower temperatures due to the fact that no ratios are available to adequately constrain the low-temperature slope.

When introducing higher-order polynomials (orders $>5$ ), the DEMs of both stars start to develop two-peaked structures with maxima at $\log T \approx 6.2-6.4$ and 6.8-7.2. This would correspond to a low- and a high temperature component, where the first one causes the strong oxygen line fluxes, and the hotter component is related to the typical active $\mathrm{M}$ dwarf corona and the observed flares. However, the DEMs constructed with higher-order polynomials are often unstable, and the location and strength of certain structures often depends only on a single line flux measurement. Thus we cannot be certain about the reality of these 

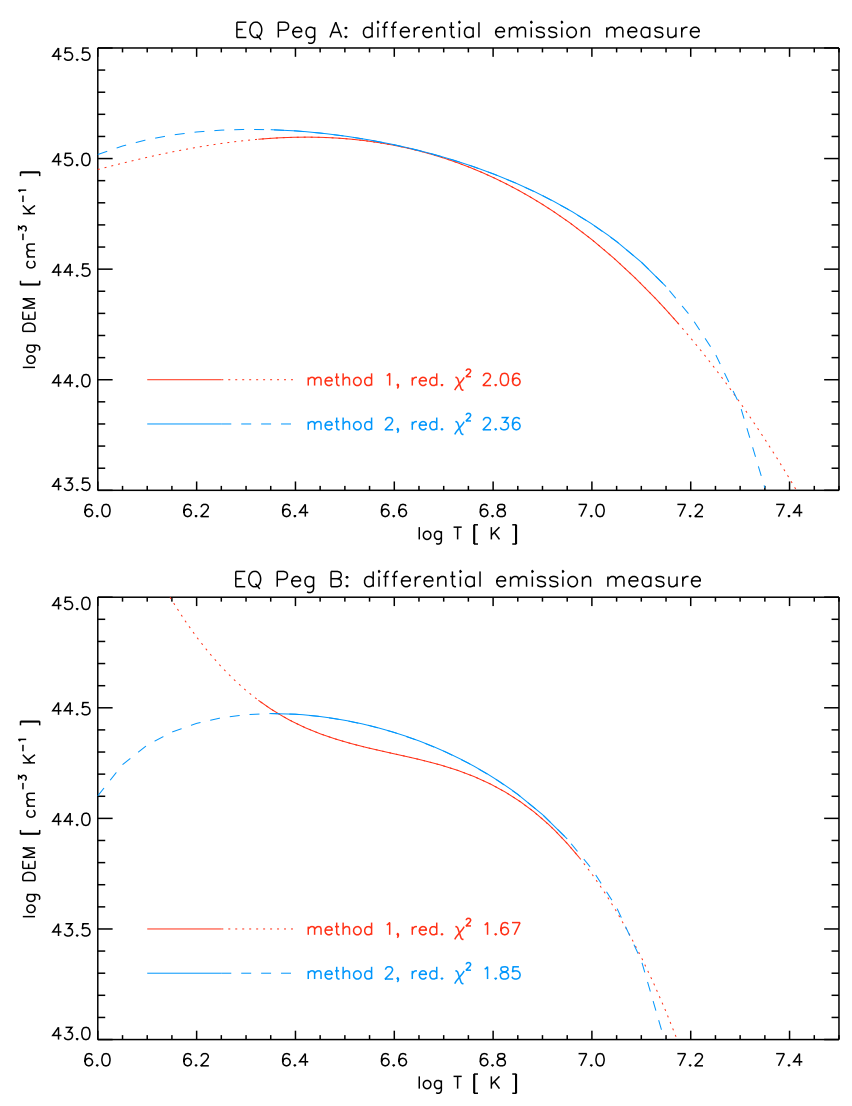

Fig. 6. Differential emission measures of EQ Peg A (top) and B (bottom) for the total observation time (i.e. flare and quiescence).

structures, and we prefer the "simpler" DEMs with fewer free parameters.

Next, we used the DEMs from these two approaches for the two stars to predict line fluxes. Since the DEMs are determined independently of the abundances and are normalized to the continuum, any discrepancies between predictions and measurements of line fluxes must be due to different abundances than those assumed in predicting the line fluxes from a given DEM (here Asplund et al. 2005).

In Table 5 we list our results for the abundances with statistical uncertainties derived from the measurement uncertainties of the line fluxes; note that the quoted errors only reflect the statistical precision of the line flux measurements, but not the accuracy of the DEM nor the uncertainties from the continuum flux nor any errors arising from the atomic physics parameters used. The errors in the continuum amount to $2.5 \%$ and $8.2 \%$ for EQ Peg A and $\mathrm{B}$, respectively, and affect only the absolute abundance level. While we experience great uncertainties in determining the exact shape of the DEM, the determination of abundances seems to be only relatively little affected. Polynomials of different orders always give abundances consistent within the errors and the abundances derived with method 1 are always consistent with those obtained from method 2. Similarly, Ness \& Jordan (2008) performed a detailed emission measure analysis of $\epsilon$ Eridani, including ionization stages from many different ions. Close to the peak, their emission measure distribution agrees with that by Sanz-Forcada et al. (2004), but is more accurate at low temperatures. In spite of the differences, both find similar abundances. In general, the derived abundances turn out to be quite insensitive to changes in the shape of the DEM, polynomials of different orders always give abundance value consistent within the errors.
Additionally, the abundances derived with method 1 are always consistent with those obtained from method 2. Liefke \& Schmitt (in preparation) assess that the uncertainties on the abundances introduced by uncertainties on the shape of the DEM do not exceed $5 \%$.

In Table 5 we show that the absolute abundances relative to Asplund et al. (2005) of EQ Peg A and B are roughly consistent for all elements, yielding a low metallicity compared to solar. This is typical for active M dwarfs (compare e.g. with the values derived by Robrade \& Schmitt 2005, from global fitting approaches to CCD X-ray spectra). Nitrogen, oxygen, and neon may be somewhat more abundant in the A component, however, not at a statistically significant level; also note that the uncertainties for $\mathrm{N}$ and $\mathrm{O}$ are quite large. While the high-FIP element neon is about solar, the low-FIP element iron shows the lowest abundance value, and the other elements also follow the typical inverse FIP effect.

The computed abundances and DEMs can now be used to model the spectra of EQ Peg A and B. We list the calculated theoretical line fluxes together with the measured fluxes in Table 2.

\subsection{Emission measure-independent abundances}

Given the ill-posed nature of the DEM determinations and the resulting uncertainties in the DEM distributions, we also investigated emission measure-independent abundance determinations. The strongest lines in stellar coronal X-ray spectra (and thus easy to measure even in spectra with low signal) originate from the H-like and He-like lines of carbon, nitrogen, oxygen, neon, magnesium, and silicon, as well as Ne-like Fe XVII. In Fig. 7 we show the line emissivities for these ions as a function of temperature. While these lines are formed over a broad temperature range, their emissivity peaks are relatively narrow. Those lines for which the line contribution functions have similar peak formation temperatures yield a similar temperature dependence. Ratios of their line fluxes depend only weakly on temperature, but strongly on the abundance ratio of the respective elements. Already Acton et al. (1975) proposed to determine the solar coronal $\mathrm{Ne} / \mathrm{O}$ abundance ratio from the ratio of the measured fluxes of the Ne IX resonance and O VIII Ly $\alpha$ lines. This method yields a comparably high precision (because of smaller measurement uncertainties), and also a higher accuracy (because no systematic uncertainties from the DEM are introduced). Recent examples of such approaches were presented by Schmitt \& Ness (2002) and Drake \& Testa (2005).

While for a given element, the peak formation temperature of the $\mathrm{H}$-like ion always exceeds that of the corresponding He-like ion, the peak formation temperatures of the H-like and He-like ions shift to higher temperature with increasing atomic mass. Therefore, pairs of H-like and He-like ions of elements with different atomic mass yield similar peak formation temperatures, and their contribution functions have similar shapes. Further examples for such pairs are the H-like lines and He-like lines of carbon and nitrogen, nitrogen and oxygen, neon and magnesium, and magnesium and silicon respectively (Parkinson 1977). However, these ratios are not perfectly temperature-independent, and Drake \& Testa (2005) have refined Acton's method by computing a linear combination of Ne X Ly $\alpha$ and the Ne IX $r$ line in order to construct a new contribution function of $\mathrm{Ne}$ lines that is more similar in shape to the O VIII Ly $\alpha$ line.

This suggests to ask in general, which linear combinations of strong lines without significant blends (i.e. hydrogen- and helium-like lines and the strongest lines of Fe XVII as mentioned above) of two certain elements yield the smallest temperature 


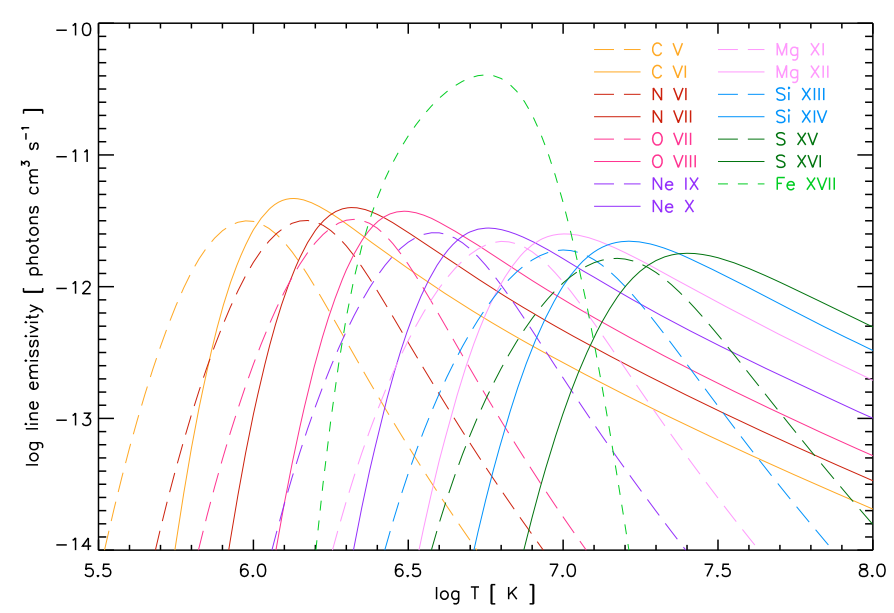

Fig. 7. Line emissivity curves for different $\mathrm{H}$-like and He-like ions, and Fe XVII at $15.01 \AA$ from CHIANTI 5.2 (Landi et al. 2006) with the ionization balance from Mazzotta et al. (1998).

residuals in their normalized contribution functions. We thus write for the abundance ratio of two elements with respective abundances $A_{1}$ and $A_{2}$

$\frac{A_{1}}{A_{2}}=\frac{f\left(A_{1}^{Z-1}\right)+C_{1} \cdot f\left(A_{1}^{Z}\right)}{C_{2} \cdot f\left(A_{2}^{Z-1}\right)+C_{3} \cdot f\left(A_{2}^{Z}\right)}$,

with $f\left(A_{1}^{Z-1}\right)$ and $f\left(A_{2}^{Z-1}\right)$ denoting the measured line fluxes $f$ of the He-like resonance lines and $f\left(A_{1}^{Z}\right)$, and $f\left(A_{2}^{Z}\right)$ the corresponding H-like Ly $\alpha$ lines of two elements $A_{1}$ and $A_{2}$, respectively, and

$\frac{A}{A_{\mathrm{Fe}}}=\frac{C_{4} \cdot\left(f\left(A^{Z-1}\right)+C_{5} \cdot f\left(A^{Z}\right)\right)}{\sum f(\mathrm{Fe} \text { XVII })}$

where $\sum f(\mathrm{Fe}$ XVII $)$ corresponds to $f(\mathrm{Fe}$ XVII $15.01 \AA)+$ $f($ Fe XVII $16.78 \AA)+f($ Fe XVII 17.05 $\AA)+f($ Fe XVII 17.09 $\AA)$, to determine relative abundance ratios.

To obtain the coefficients $C_{1}, C_{2}, C_{3}, C_{4}$, and $C_{5}$, we performed a minimization of the temperature residuals of the corresponding linear combination of the theoretical emissivities $\epsilon$ for the involved lines over a given temperature range:

$$
\begin{aligned}
\chi^{2}= & \sum_{i}\left(\epsilon_{i}\left(A_{1}^{Z-1}, T_{i}\right)+C_{1} \cdot \epsilon_{i}\left(A_{1}^{Z}, T_{i}\right)\right. \\
& \left.-C_{2} \cdot \epsilon_{i}\left(A_{2}^{Z-1}, T_{i}\right)-C_{3} \cdot \epsilon_{i}\left(A_{2}^{Z}, T_{i}\right)\right)^{2}
\end{aligned}
$$

for the coefficients in Eq. (6), and

$\chi^{2}=\sum_{i}\left(\epsilon_{i}\left(A^{Z-1}, T_{i}\right)+C_{5} \cdot \epsilon_{i}\left(A^{Z}, T_{i}\right)-\frac{1}{C_{4}} \cdot \sum \epsilon_{i}\left(\mathrm{Fe} \text { XVII }, T_{i}\right)\right)^{2}(9)$

for the coefficients in Eq. (7).

In Table 6 we list these coefficients for the temperature range where the line emissivities of all involved lines exceed $1 \%$ of their peak emissivity. In the given form, the coefficients convert the measured line fluxes in photon flux units to absolute abundance ratios, i.e. independent from any set of solar photospheric abundances; we used line emissivities from CHIANTI 5.2 (Landi et al. 2006).

There are still residuals in temperature for these linear combinations, and their amplitudes differ for each ratio. We found
Table 6. Coefficients for Eqs. (6) and (7) to obtain temperatureindependent abundance ratios.

\begin{tabular}{lccccc}
\hline \hline Ratio & $C_{1}$ & $C_{2}$ & $C_{3}$ & $C_{4}$ & $C_{5}$ \\
\hline $\mathrm{N} / \mathrm{C}$ & +0.13 & -0.07 & +0.73 & - & - \\
$\mathrm{O} / \mathrm{N}$ & +0.30 & +0.01 & +0.93 & - & - \\
$\mathrm{Ne} / \mathrm{O}$ & +0.02 & -0.17 & +0.69 & - & - \\
$\mathrm{Mg} / \mathrm{Ne}$ & +0.18 & -0.08 & +0.87 & - & - \\
$\mathrm{Si} / \mathrm{Mg}$ & +0.32 & +0.05 & +0.86 & - & - \\
$\mathrm{S} / \mathrm{Si}$ & +0.42 & +0.15 & +0.85 & - & - \\
$\mathrm{Ne} / \mathrm{Fe}$ & - & - & - & +34.71 & +0.46 \\
$\mathrm{Mg} / \mathrm{Fe}$ & - & - & - & +67.73 & -0.30 \\
\hline
\end{tabular}

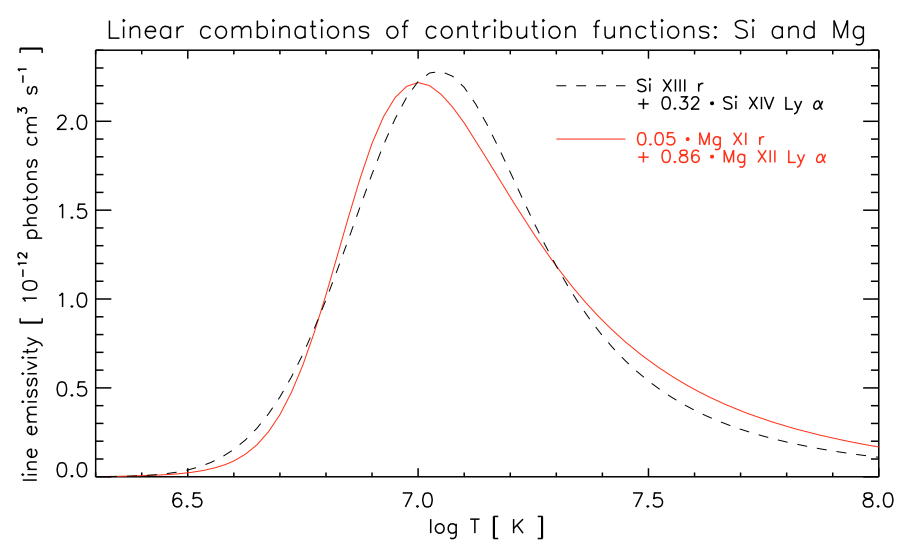

Fig. 8. Best-fit linear combination of the contribution functions of the $\mathrm{H}$-like Ly $\alpha$ and He-like resonance lines of silicon and magnesium.

the lowest residuals for the $\mathrm{Si} / \mathrm{Mg}$ ratio and the largest ones for the $\mathrm{Ne} / \mathrm{O}$ ratio. In Fig. 8 we illustrate the best-fit linear combination of emissivities that yield the $\mathrm{Si} / \mathrm{Mg}$ abundance ratio, and for the Ne/O ratio we refer to Fig. 3 of Liefke \& Schmitt (2006), who had used the same coefficients as listed here to calculate the $\mathrm{Ne} / \mathrm{O}$ abundance ratio of $\alpha$ Centauri. The first six ratios involve only $\mathrm{H}$-like Ly $\alpha$ and He-like resonance lines. However, the S/Si ratio should only be used when the sulfur lines can be measured with reasonable signal-to-noise in Chandra HETGS spectra. Fe XVII can be matched with linear combinations of hydrogen- and helium-like lines of neon or magnesium. The residuals are much smaller for $\mathrm{Mg}$, however, the $\mathrm{Mg}$ lines are often weak, introducing large measurement uncertainties. Especially the weak Mg XI line carries a large weight, and for the determination of $\mathrm{Fe}$ abundances we prefer the $\mathrm{Ne} / \mathrm{Fe}$ ratio despite its larger residuals.

The coefficients of the linear combinations as listed in Table 6 can be adjusted in some cases where certain line fluxes are not available (e.g. the $\mathrm{CV}$ lines can only be measured with the Chandra LETGS), or are extremely weak (e.g. Mg XI or $\mathrm{N}$ VI, depending on temperature). The line fluxes $f\left(A_{1}^{Z-1}\right)$ and $f\left(A_{2}^{Z}\right)$ with $Z\left(A_{1}\right)>Z\left(A_{2}\right)$ provide the major constituents of the linear combinations for the ratios not involving $\mathrm{Fe}$.

For example, the NVI resonance line is often weak in stars with hot coronae and is difficult to measure in Chandra MEG spectra, however, this line contributes much less to the nitrogen linear combination in the $\mathrm{O} / \mathrm{N}$ ratio than the $\mathrm{N}$ VII Ly $\alpha$ line (i.e. $C_{2}$ is much smaller than $C_{3}$ ). We readjusted $C_{1}$, and $C_{3}$ assuming the fixed value $C_{2}=0$ and found only marginal changes for the values of the other coefficients listed in Table 6. Although the value of $C_{1}$ is also quite low for the $\mathrm{Ne} \mathrm{X}$ Ly $\alpha$ line in the $\mathrm{Ne} / \mathrm{O}$ ratio, the situation is somewhat different. If we readjust the other coefficient after setting $C_{1}=0$, the 
Table 7. Abundance ratios (relative to Asplund et al. 2005) applying temperature structure-independent linear combinations of coronal emission lines. Values given in brackets for EQ Peg A correspond to the quiescent state.

\begin{tabular}{cccc}
\hline \hline Ratio & \multicolumn{2}{c}{ EQ Peg A } & EQ Peg B \\
\hline $\mathrm{O} / \mathrm{N}$ & $0.83 \pm 0.20$ & $(1.00 \pm 0.29)$ & $0.81 \pm 0.72$ \\
$\mathrm{Ne} / \mathrm{O}$ & $2.48 \pm 0.31$ & $(2.02 \pm 0.16)$ & $2.18 \pm 0.41$ \\
$\mathrm{Mg} / \mathrm{Ne}$ & $0.20 \pm 0.02$ & $(0.19 \pm 0.02)$ & $0.32 \pm 0.07$ \\
$\mathrm{Si} / \mathrm{Mg}$ & $2.52 \pm 0.21$ & $(2.56 \pm 0.25)$ & $2.05 \pm 0.44$ \\
$\mathrm{~S} / \mathrm{Si}$ & $1.26 \pm 0.20$ & - & - \\
$\mathrm{Ne} / \mathrm{Fe}$ & $7.30 \pm 0.64$ & $(5.99 \pm 0.34)$ & $5.45 \pm 0.78$ \\
$\mathrm{Mg} / \mathrm{Fe}$ & $1.27 \pm 0.17$ & $(1.09 \pm 0.19)$ & $1.63 \pm 0.43$ \\
\hline
\end{tabular}

Table 8. Abundance ratios relative to Asplund et al. (2005) of EQ Peg A and $\mathrm{B}$, determined from the absolute abundances listed in Table 5.

\begin{tabular}{ccccc}
\hline \hline & \multicolumn{2}{c}{ EQ Peg A } & \multicolumn{2}{c}{ EQ Peg B } \\
Ratio & Method 1 & Method 2 & Method 1 & Method 2 \\
\hline $\mathrm{O} / \mathrm{N}$ & $1.00 \pm 0.25$ & $1.03 \pm 0.26$ & $0.94 \pm 0.94$ & $1.30 \pm 1.24$ \\
$\mathrm{Ne} / \mathrm{O}$ & $2.71 \pm 0.31$ & $2.68 \pm 0.32$ & $6.20 \pm 3.75$ & $3.19 \pm 0.94$ \\
$\mathrm{Mg} / \mathrm{Ne}$ & $0.19 \pm 0.01$ & $0.19 \pm 0.02$ & $0.30 \pm 0.05$ & $0.33 \pm 0.06$ \\
$\mathrm{Si} / \mathrm{Mg}$ & $2.32 \pm 0.14$ & $2.30 \pm 0.25$ & $1.93 \pm 0.37$ & $1.93 \pm 0.39$ \\
$\mathrm{~S} / \mathrm{Si}$ & $1.25 \pm 0.32$ & $1.26 \pm 0.46$ & - & - \\
$\mathrm{Ne} / \mathrm{Fe}$ & $6.00 \pm 0.73$ & $5.94 \pm 0.51$ & $4.65 \pm 0.58$ & $4.88 \pm 0.71$ \\
$\mathrm{Mg} / \mathrm{Fe}$ & $1.15 \pm 0.13$ & $1.11 \pm 0.13$ & $1.40 \pm 0.24$ & $1.59 \pm 0.30$ \\
\hline
\end{tabular}

residuals are much larger, especially at high temperatures where the emission measure distributions of active stars usually have their maximum.

Finally, the coefficients can be re-computed for the sum of the He-like resonance, intercombination, and forbidden lines for cases where these lines can not be resolved (e.g. Si XIII with the Chandra LETGS).

We applied Eqs. (6) and (7), with the coefficients from Table 6 multiplied by the solar photospheric abundances of Asplund et al. (2005), to the line fluxes measured from the spectra of EQ Peg A (including and excluding periods of flare activity) and of EQ Peg B and list the resulting abundance ratios in Table 7.

Within the uncertainties, the derived abundance ratios are consistent for all three datasets. Only the $\mathrm{Ne} / \mathrm{Fe}$ ratio is conspicuously lower in the quiescent spectrum of EQ Peg A compared to the total spectrum, and again lower for the more inactive EQ Peg B. Flare- or activity-related changes in the abundance ratios cannot be determined from count statistics; however, if real, the differences may be caused by a decreased iron abundance level or a higher neon abundance on the more active A component, especially during flares.

In Table 8 we list the abundance ratios formed from the absolute values listed in Table 5 for comparison. They are consistent within the errors with the values in Table 7 , but the ratios obtained from the emission measure-independent method are more robust and have lower uncertainties.

\section{EQ Peg in the context of active M dwarfs}

In order to put EQ Peg into the context of other similar latetype stars, we extracted five additional Chandra HETGS spectra of active M dwarfs for comparison with our results; our sample thus contains YY Gem, AU Mic, EV Lac, AD Leo, and Proxima Cen in addition to EQ Peg A and B. Most of these archival observations have been included in many studies (e.g. Ness et al. 2003c, 2004; Testa et al. 2007), the X-ray lightcurves and spectra of AU Mic, EV Lac, and Proxima Cen are shown by Testa et al. (2004). While the two stars in the binary EQ Peg are co-eval, we are now dealing with a range of different ages, rotation periods, and metallicity. In Table 9 we summarize for each star their spectral type, distance (from the SIMBAD database), age estimates and rotation periods from the literature together with observation ID, exposure time, and X-ray luminosity obtained from integration of the dispersed photons over the range $2-25 \AA$. The bolometric luminosity for $L_{\mathrm{X}} / L_{\mathrm{bol}}$ has been calculated according to Kenyon \& Hartmann (1995). While the X-ray luminosity decreases along the sequence of spectral types, $L_{\mathrm{X}} / L_{\mathrm{bol}}$ is in the saturation regime of $\approx-3.3$ for all stars except Proxima Cen.

YY Gem is an M0.5 companion of the Castor AB system. YY Gem itself is an eclipsing binary consisting of two almost identical early $\mathrm{M}$ dwarfs with an orbital period of 0.814 days at an inclination of 86.2 , separated by $3.88 R_{\odot}$. From eclipse mapping techniques based on an XMM-Newton observation, Güdel et al. (2001) found similar activity levels on both components, but concentrated in compact active regions at small scale heights, i.e. inter-binary loops as discussed for RS CVn systems are rather unlikely. This is confirmed by Stelzer et al. (2002), who modelled a flare in a simultaneous XMM-Newton and Chandra LETGS observation. Several strong flares occur also in the two HETGS observations discussed here. The young ( $\approx 10 \mathrm{Myr})$ star AU Mic, spectral type M1, has the highest X-ray luminosity within $10 \mathrm{pc}$ to the Sun and has thus been extensively observed in X-rays and in the EUV. AU Mic shows a strong level of variability on all time scales and a high flare rate. From the HETGS dataset used here, Linsky et al. (2002) computed an emission measure distribution with a peak temperature of $\log T \approx 6.8$ and subsolar abundances with a pronounced inverse FIP effect. Magee et al. (2003) found similar values for the abundances based on an XMM-Newton observation. The Chandra HETGS observation of the M3.5 star $\mathrm{EV}$ Lac is part of a multiwavelength campaign and has been discussed in detail by Osten et al. (2005, 2006). Several flares occurred towards the end of the observation, and Osten et al. (2005) compared line ratios with different FIP dependencies during flares and quiescence and find a slight increase for low FIP/high FIP ratios during flares. Osten et al. (2006) derive a subsolar abundance level and an inverse FIP effect from an emission measure distribution of the quiescent state, with values consistent with an XMM observation analyzed by Robrade \& Schmitt (2005). Both EV Lac and the M4.5 star AD Leo are young stars from the galactic disk population. AD Leo is another a well-known flare star (e.g. Hawley et al. 2003), however, no larger flares have occurred during the Chandra HETGS observation discussed here. This dataset was analyzed by Maggio \& Ness (2005), with the focus on density diagnostics. Maggio et al. (2004) found a mild inverse FIP bias based on two Chandra LETGS observations, which is roughly consistent with the results obtained by Robrade \& Schmitt (2005) and from an XMM-Newton observation. van den Besselaar et al. (2003) analyzed the same data. While Robrade \& Schmitt and van den Besselaar et al. determined an overall subsolar abundance level, Maggio et al. found values much higher than for the solar photosphere, however with a large uncertainty in the overall normalization. EQ Peg A, EV Lac, and AD Leo have comparable X-ray luminosities. On the basis of a common origin with the $\alpha$ Centauri system, Proxima Cen can be considered older than the Sun. With a spectral type of M5.5, it is also the latest star in our sample. Its X-ray luminosity is comparable to the Sun, more than two orders of magnitude lower than that of AU Mic. Nevertheless, Proxima Cen is also a well-known flare star. 
Table 9. Properties of active M dwarfs observed with Chandra HETGS. X-ray luminosities are computed from the total background-subtracted counts contained in the MEG spectra from 2-25 A. Values given in brackets correspond to the quiescent state.

\begin{tabular}{|c|c|c|c|c|c|c|c|c|c|c|c|}
\hline \multirow{2}{*}{$\begin{array}{l}\text { Star } \\
\text { YY Gem }\end{array}$} & \multirow{2}{*}{$\begin{array}{c}\begin{array}{c}\text { Spectral } \\
\text { Type }\end{array} \\
\text { M } 0.5\end{array}$} & \multirow{2}{*}{$\begin{array}{c}\begin{array}{c}\text { Distance } \\
{[\mathrm{pc}]}\end{array} \\
15.27\end{array}$} & \multirow{2}{*}{$\begin{array}{c}\begin{array}{c}\text { Age } \\
{[\mathrm{Myr}]}\end{array} \\
370^{1}\end{array}$} & \multirow{2}{*}{$\begin{array}{c}\begin{array}{c}P_{\text {rot }} \\
{[\mathrm{d}]}\end{array} \\
0.814^{5}\end{array}$} & \multirow{2}{*}{$\begin{array}{c}\text { ObsID } \\
8504 / 8509\end{array}$} & \multicolumn{2}{|c|}{$\begin{array}{c}\text { Exposure time } \\
{[\mathrm{ks}]}\end{array}$} & \multicolumn{2}{|c|}{$\overline{l o g} L_{X}$} & \multicolumn{2}{|c|}{$\overline{\log L_{X} / L_{\mathrm{bol}}}$} \\
\hline & & & & & & 136.0 & $(81.5)$ & 29.63 & $(29.43)$ & -3.17 & $(-3.37)$ \\
\hline AU Mic & M 1 & 9.94 & $12^{2}$ & $4.865^{6}$ & 17 & 58.8 & (47.4) & 29.19 & $(29.13)$ & -3.48 & $(-3.54)$ \\
\hline EQ Peg A & M 3.5 & 6.25 & $950^{3}$ & $\left(1.066^{7}\right)$ & $8453 / 8484 / 8485 / 8486$ & 98.5 & $(92.8)$ & 28.71 & (28.67) & -3.19 & $(-3.23)$ \\
\hline EV Lac & M 3.5 & 5.05 & $45^{3}$ & $4.376^{8}$ & 1885 & 100.0 & $(87.3)$ & 28.63 & $(28.51)$ & -3.10 & $(-3.22)$ \\
\hline AD Leo & M 4.5 & 4.69 & $470^{3}$ & $2.7 / 14^{9,10}$ & 2570 & 45.2 & - & 28.54 & - & -3.36 & - \\
\hline EQ Peg B & M 4.5 & 6.25 & $950^{3}$ & $\left(1.066^{7}\right)$ & $8453 / 8484 / 8485 / 8486$ & 98.5 & - & 27.89 & - & -3.39 & - \\
\hline Proxima Cen & M 5.5 & 1.30 & $5800^{4}$ & $83.5^{10}$ & 2388 & 42.4 & $(35.7)$ & 26.85 & $(26.60)$ & -3.96 & $(-4.21)$ \\
\hline
\end{tabular}

${ }^{1}$ Torres \& Ribas (2002); ${ }^{2}$ Zuckerman et al. (2001); ${ }^{3}$ Parsamyan (1995); ${ }^{4}$ Yildı (2007); ${ }^{5}$ Kron (1952); ${ }^{6}$ Torres et al. (1972); ${ }^{7}$ Norton et al. (2007); ${ }^{8}$ Pettersen et al. (1992); ${ }^{9}$ Spiesman \& Hawley (1986); ${ }^{10}$ Mahmoud (1993); ${ }^{11}$ Benedict et al. (1998).

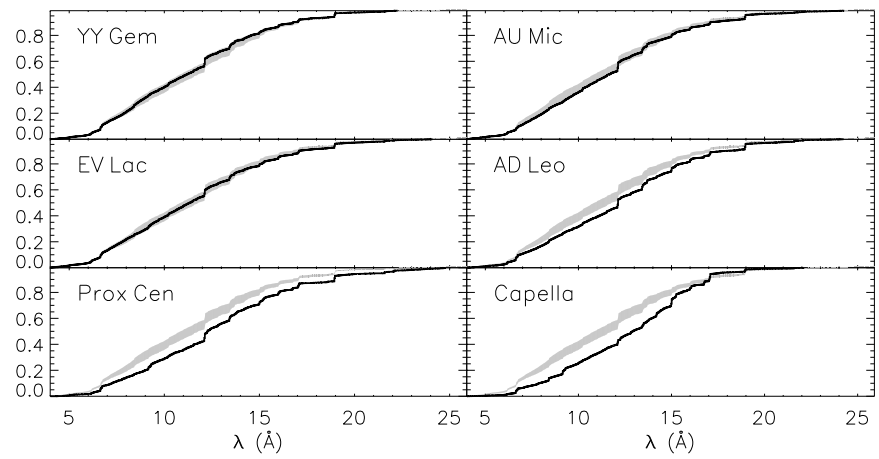

Fig. 9. Cumulative distribution of counts in the quiescent spectra of the reference star Capella and other M dwarfs observed with the Chandra HETGS. The area between the curves for EQ Peg A and B is indicated in shades of gray for comparison.

Güdel et al. (2002a) and Güdel et al. (2004) discussed a largeamplitude long-duration flare observed with XMM-Newton. Güdel et al. (2004) and Nordon \& Behar (2008) derived emission measure distributions for the different phases of the flare and found abundance ratios relative to iron to stay roughly constant, but with an overall tendency to an inverse FIP effect in the absolute level. Two flares are also included in the HETGS observation analyzed here.

In order to obtain self-consistent results, we applied the same data reduction and analysis techniques as described above to the spectra of the other four sample stars. However, even with typical flare rates and amplitudes for active $\mathrm{M}$ dwarfs known, the occurrence of individual flares is randomly distributed, and each X-ray observation is a snapshot that is not necessarily representative of the typical flaring behavior of the observed star. We filtered the observation for quiescent-only emission and include the quiescent luminosities in brackets in the last column of Table 9.

We also computed the cumulative count spectra for the other five spectra as that shown in Fig. 3 for EQ Peg. In Fig. 9 we show these spectra for the quiescent states of the five stars as labeled in the legends, together with the well-exposed reference spectrum of Capella. For comparison, we included the cumulative spectra of EQ Peg A and B, bracketing the gray-shaded areas. In these plots, YY Gem, AU Mic and EV Lac are similar to the EQ Peg system, with YY Gem very similar to EQ Peg A, while AU Mic has stronger neon lines than EQ Peg, leading to larger steps in the cumulative distribution. AD Leo is more similar to EQ Peg B, and Proxima Cen is even softer. All seven M dwarfs differ considerably from Capella.
We applied our DEM-independent abundance determination using Eq. (6) with the coefficients listed in Table 6 to the total and quiescent-only spectra of AU Mic, EV Lac, AD Leo, and Proxima Cen and list the results in Table 10. These numbers have to be compared to the relative abundances for EQ Peg A and B listed in Table 7. For comparison of our results with other abundance determinations for our sample stars, we transformed the values determined by Robrade \& Schmitt (2005), Güdel et al. (2001), Linsky et al. (2002), Magee et al. (2003), Osten et al. (2006), Maggio et al. (2004), and Güdel et al. (2004) to the reference set of solar photospheric abundances from Asplund et al. (2005) and found good agreement within the errors. Only for neon we found a slight tendency of overestimating the abundance, as the blends to the neon lines may not always have been taken properly into account.

For all seven stars, the $\mathrm{Ne} / \mathrm{O}$ ratio is enhanced by about a factor of 2, which is well-consistent with what Drake \& Testa (2005) found for their sample of active stars. Si/Mg is also enhanced by a factor of about 2 , while $\mathrm{Mg} / \mathrm{Ne}$ is depleted by factors from 3 (EQ Peg B and Proxima Cen) up to 10 (AU Mic). The values for $\mathrm{O} / \mathrm{N}$ range from $0.3-1.0$ times the solar level, partly with large uncertainties. The $\mathrm{Ne} / \mathrm{Fe}$ ratio is clearly increased, by factors ranging from 4.5 (Proxima Cen) to 9 (AU Mic), while the $\mathrm{Mg} / \mathrm{Fe}$ ratio is about solar. In Fig. 10 we give a graphical representation of the numbers listed in Tables 7 and 10 from the quiescent data.

$\mathrm{O} / \mathrm{N}$ and $\mathrm{Ne} / \mathrm{O}$ can be considered as high FIP/high FIP ratios, and within the errors, these ratios do not change with spectral type. No trends can also be identified for the low FIP/low FIP ratios $\mathrm{Mg} / \mathrm{Fe}$ and $\mathrm{Si} / \mathrm{Mg}$. The low $\mathrm{FIP} /$ high FIP ratio $\mathrm{Mg} / \mathrm{Ne}$ on the other hand clearly increases towards later spectral type, while the high FIP/low FIP ratio $\mathrm{Ne} / \mathrm{Fe}$ decreases.

$\mathrm{Mg} / \mathrm{Ne}$ and $\mathrm{Ne} / \mathrm{Fe}$ are sensitive to a possible FIP bias, and both are anomalously low and high, respectively, for all stars in our sample, pointing towards an inverse FIP effect. This result holds for both total and quiescent-only spectra. Since we only have relative abundances, it is unclear whether high-FIP elements are overabundant or low-FIP elements are underabundant. A low $\mathrm{Mg} / \mathrm{Ne}$ ratio may be caused by an enhancement of neon, as supported by the increased $\mathrm{Ne} / \mathrm{O}$ ratios, but also by a low $\mathrm{Mg}$ abundance, which would match the enhanced $\mathrm{Si} / \mathrm{Mg}$ ratios, and so on.

The trends observed for $\mathrm{Ne} / \mathrm{Fe}$ and $\mathrm{Mg} / \mathrm{Ne}$ are roughly independent of the occurrence of flares. This confirms previous findings that strength of the inverse FIP effect scales with the general activity level of a star (Güdel et al. 2002b; Telleschi et al. 2005), as activity (in terms of X-ray luminosity) decreases with increasing spectral type. While the behavior of the $\mathrm{Ne} / \mathrm{Fe}$ and 
Table 10. Abundance ratios of other M dwarfs observed with the Chandra HETGS. Values obtained for the quiescent state are given in brackets.

\begin{tabular}{cccccccccc}
\hline \hline Ratio & \multicolumn{2}{c}{ YY Gem } & \multicolumn{2}{c}{ AU Mic } & \multicolumn{2}{c}{ EV Lac } & AD Leo & Proxima Cen \\
\hline $\mathrm{O} / \mathrm{N}$ & $0.62 \pm 0.12$ & $(0.57 \pm 0.14)$ & $0.48 \pm 0.09$ & $(0.48 \pm 0.11)$ & $0.53 \pm 0.10$ & $(0.76 \pm 0.15)$ & $0.60 \pm 0.13$ & $0.30 \pm 0.09$ & $(0.34 \pm 0.14)$ \\
$\mathrm{Ne} / \mathrm{O}$ & $1.97 \pm 0.12$ & $(2.10 \pm 0.18)$ & $2.36 \pm 0.19$ & $(2.31 \pm 0.20)$ & $2.07 \pm 0.50$ & $(1.71 \pm 0.12)$ & $2.33 \pm 0.19$ & $1.72 \pm 0.31$ & $(2.06 \pm 0.54)$ \\
$\mathrm{Mg} / \mathrm{Ne}$ & $0.18 \pm 0.02$ & $(0.16 \pm 0.02)$ & $0.11 \pm 0.01$ & $(0.12 \pm 0.02)$ & $0.24 \pm 0.02$ & $(0.22 \pm 0.02)$ & $0.21 \pm 0.02$ & $0.31 \pm 0.06$ & $(0.35 \pm 0.10)$ \\
$\mathrm{Si} / \mathrm{Mg}$ & $2.31 \pm 0.18$ & $(2.59 \pm 0.31)$ & $2.33 \pm 0.26$ & $(1.84 \pm 0.26)$ & $2.47 \pm 0.22$ & $(2.25 \pm 0.25)$ & $2.46 \pm 0.30$ & $2.48 \pm 0.28$ & $(2.50 \pm 1.28)$ \\
$\mathrm{S} / \mathrm{Si}$ & $1.27 \pm 0.18$ & $(1.46 \pm 0.33)$ & - & - & $1.82 \pm 0.33$ & - & - & - & - \\
$\mathrm{Ne} / \mathrm{Fe}$ & $7.08 \pm 0.33$ & $(7.34 \pm 0.46)$ & $8.85 \pm 0.57$ & $(9.03 \pm 0.59)$ & $5.73 \pm 0.29$ & $(5.17 \pm 0.26)$ & $6.78 \pm 0.41$ & $4.44 \pm 0.59$ & $(4.45 \pm 0.82)$ \\
$\mathrm{Mg} / \mathrm{Fe}$ & $1.23 \pm 0.19$ & $(1.24 \pm 0.24)$ & $0.84 \pm 0.18$ & $(1.03 \pm 0.26)$ & $1.28 \pm 0.15$ & $(1.28 \pm 0.15)$ & $1.13 \pm 0.19$ & $1.44 \pm 0.35$ & $(1.60 \pm 0.49)$ \\
\hline
\end{tabular}

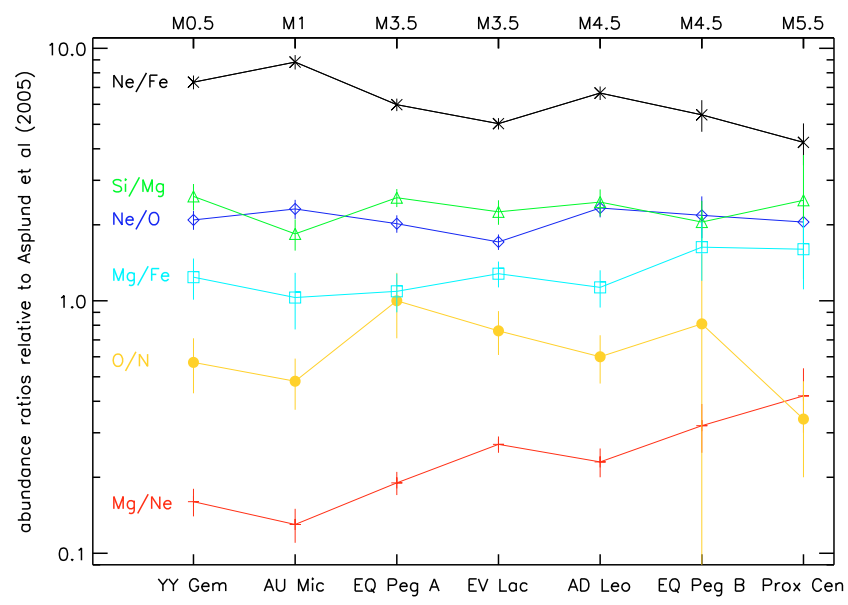

Fig. 10. Relative abundances as a function of spectral type derived from the seven M dwarfs as listed in Tables 7 and 10.

$\mathrm{Mg} / \mathrm{Ne}$ ratios in our $\mathrm{M}$ dwarf sample implies an absolute decrease of neon with later spectral type, the constant $\mathrm{Ne} / \mathrm{O}$ ratio does not fit that picture. This indicates that not only a single element like neon causes this effect, but that the inverse FIP effect in general diminishes for later M dwarfs.

\section{Discussion}

The behavior of $\mathrm{M}$ dwarf coronae shows striking changes with spectral type. The early $\mathrm{M}$ dwarfs up to a spectral type of ₹M3-M4 observed so far are strong X-ray emitters, showing permanent flaring, high coronal temperatures and a pronounced inverse FIP effect (Robrade \& Schmitt 2005). X-ray luminosity, the level and peak temperature of the emission measure, and the flaring rate decrease for later $\mathrm{M}$ dwarfs. While for earlier $M$ subclasses, it is difficult - if not impossible - to distinguish between weak flaring and quiescence, $M$ dwarfs of spectral type M5 or later show periods of quiescence interrupted by discrete flares, and their coronal temperatures decrease (Fuhrmeister et al. 2007). Very late M dwarfs (>M7) become more and more difficult to detect as quiescent X-ray sources, yet they can still produce enormous X-ray luminosities during flares. Abundance determinations are difficult for these objects, but Güdel et al. (2004) and Fuhrmeister et al. (2007) found the inverse FIP effect to be less pronounced in the M5.5 stars Proxima Cen and CN Leo. With our systematic line ratio survey, we can consolidate the trend of the inverse FIP effect in $\mathrm{M}$ dwarf coronae weakening with later spectral subclass. This is coupled to the decrease of the activity observed with later subclass in general, while $L_{\mathrm{X}} / L_{\mathrm{bol}}$ is similar and dominated by saturation effects for all our sample stars except for the latest object Proxima Cen. Apart from the precise strength of single emission lines that are mostly determined by individual abundances, high resolution X-ray spectra of $\mathrm{M}$ dwarfs have a very similar overall shape. Only the continuum level decreases with later spectral type, as lower coronal temperatures reduce the amount of true continuum emission as well as the pseudo-continuum dominated by lines of highly ionized iron located at approximately 9-17 A. The reduction of the continuum level can easily be quantified from the cumulative spectra and serves as an indicator for the average coronal temperature.

Both methods we used for our analysis, the cumulative spectra as well as the emission measure independent line ratios, can be applied to spectra with lower signal where only the strongest lines can be discerned. They are thus also suitable to determine coronal properties from the spectra of the fainter mid and late M dwarfs. Only very few grating spectra of such objects are available so far, and especially spectra of stars later than M6 would help to confirm the trend that the difference of the $\mathrm{Ne} / \mathrm{Fe}$ and $\mathrm{Mg} / \mathrm{Ne}$ ratios to solar photospheric values decreases with later spectral type. However, since the $\mathrm{Ne} / \mathrm{O}$ and $\mathrm{Si} / \mathrm{Mg}$ ratios stay enhanced by about a factor of two, there is no indication of all abundance anomalies to diminish. So at least in comparison to the saturated coronae of active M dwarfs, it is the solar photosphere which appears anomalous; and the abundances of M dwarfs seem to "converge" with later spectral subclass, but to values differing from the solar photospheric level.

The determination of reference sets of photospheric abundances of active $\mathrm{M}$ dwarfs is rather difficult, since reliable models of the stellar atmosphere including radiative transfer are required. The parameters involved in the modeling procedure, i.e. the stellar mass, effective temperature, $\log g$, and for $\mathrm{M}$ dwarfs, the influence of the stellar chromosphere and possibly the formation of dust, introduce large uncertainties in the derived abundances (Zboril \& Byrne 1998). Additionally, only the abundances of heavier elements, often only that of iron, can be determined in the photosphere, while light elements like carbon, nitrogen and oxygen or noble gases like neon that dominate the coronal spectrum leave only weak or no signatures in the photospheric spectrum.

It may be questionable to line up active $\mathrm{M}$ dwarfs exclusively based on spectral type, since other parameters, especially the age, certainly have a non-negligible influence, and age-activity relations are well-established over all wavelengths and in particular for coronal X-ray emission (Vaiana et al. 1992; Favata et al. 1994). Our ultraluminous sample star AU Mic forms a proper motion pair with the M4/M4.5 binary AT Mic, both stars are members of the $\beta$ Pictoris moving group and only about 10-12 Myr old (Zuckerman et al. 2001). Robrade \& Schmitt (2005) found AT Mic half an order of magnitude brighter in X-rays than our sample stars AD Leo, EV Lac and EQ Peg, which have similar spectral types. Stars with a common origin, like the two ZAMS stars AU Mic and AT Mic, or the coeval components of the binary EQ Peg thus provide a testbed 
independent of such biases. The M3.5 and M4.5 components of EQ Peg differ by one spectral subclass, but the X-ray luminosity of the M4.5 secondary is considerably lower, which we can attribute to a fairly reduced amount of coronal emission measure, but at temperatures similar to those found on the primary. Yet, in terms of $L_{X} / L_{\text {bol }}$, both stars are in the saturation regime, and thus again comparable. Similar considerations apply to a larger sample of different ages, ranging from a few Myr (AU Mic) to almost one Gyr (EQ Peg); the cumulative spectra of very young stars like AU Mic or EV Lac are very similar to the much older EQ Peg system. Proxima Cen is also by far the oldest object, and the one with the longest rotation period. It is the only star from our sample where $L_{\mathrm{X}} / L_{\mathrm{bol}}$ deviates from the saturation level, yet it still fits the picture of the other saturated stars in terms of abundance trends. Here we probably see the wellestablished relationships between X-ray luminosity and age or, related via spin-down with age, rotation period/rotational velocity and X-ray luminosity (Pizzolato et al. 2003; Reiners 2007) or other activity indicators like $\mathrm{H} \alpha$ or $\mathrm{Ca}$ II emission at work. The age of the other sample stars is less than $1 \mathrm{Gyr}$ and their rotation periods are typically only a few days. The sample of $\mathrm{M}$ dwarfs observed with high spectral resolution in X-rays is - by necessity - biased towards the more luminous younger objects, and it would be crucial to examine how further old and inactive objects like e.g. Barnard's star fit into that picture.

During quiescence, coronal densities on active stars typically range between $10^{10}-10^{11} \mathrm{~cm}^{-3}$ for the low-temperature plasma $(\approx 2 \mathrm{MK})$ traced by the O VII triplet (Ness et al. 2003a), and between $10^{11}-10^{12} \mathrm{~cm}^{-3}$ for Ne IX $(\approx 4 \mathrm{MK})$. Significantly higher values have so far only been observed during huge flares (Güdel et al. 2002a; Liefke et al. in preparation), but in general, coronal densities tend to increase during periods of increased activity and lower amplitude flaring (e.g. Maggio \& Ness 2005; Fuhrmeister et al. 2007). Mitra-Kraev \& Ness (2006) thus developed a statistical approach that showed that the different $f / i$ ratios observed during flares and quiescence on EV Lac are actually highly significant.

Flares can considerably change the X-ray properties of active $\mathrm{M}$ dwarf stars. The flare plasma often dominates the coronal emission, although the dimensions of the flaring loops seldomly reach the size of the star itself, and the flaring region typically covers only a small fraction of the stellar surface. The plasma temperature and emission measure can increase by more than an order of magnitude during the strongest flares, which subsequently results in an enhanced level of continuum emission. The bulk of line emission is dominated by high-temperature ions. Based on current flare models, the flare plasma is largely composed of evaporated chromospheric or photospheric material filling the loop. In this context, abundance gradients between the different layers of the atmosphere, like the FIP effect observed on the Sun, become important. Nordon \& Behar (2007) and Nordon \& Behar (2008) investigated changes in the FIP bias during several larger flares on a sample of different active stars. For the very active stars that show an inverse FIP effect during quiescence, they found abundance ratios of the scheme high FIP/low FIP like $\mathrm{Ne} / \mathrm{Fe}$ to decrease during flares, i.e. the inverse FIP effect turns into a solar-like pattern or even a FIP effect. This is in good agreement with the trends we find in our M dwarf sample. Thus abundance gradients, resulting in a different composition of the evaporated flare plasma, seem to be a common feature, and in contradiction to the corona, the underlying abundances of the lower atmospheric layers in active $\mathrm{M}$ dwarfs approach a more solar photospheric like pattern. This finding is also supported by Liefke et al. (in preparation), who found the coronal iron abundance of the M5.5 dwarf CN Leo to increase by a factor of two to the solar photospheric level during a large flare.

To what extent flares do have to be considered as the "usual" behavior of a stellar corona? Early $\mathrm{M}$ dwarfs are in a state of permanent flaring, and even during phases of apparent quiescence, the underlying basal coronal flux is difficult to disentangle from unresolved weaker flares. While larger flares can be separated, the characteristic flickering of early $\mathrm{M}$ dwarfs has to be taken as their typical "quasi-quiescent" behavior. The average coronal temperatures and densities of active stars will lower when the data is restricted to this quiescent state. However, we do not find that the flares included in the total datasets of our seven sample stars introduce large changes in the observed abundance ratios. Since the data do not include huge flares, this indicates a smooth transition between the quasi-quiescent state and intermediate flares, which supports the assumption that the unresolved flickering is indeed composed of smaller flares. When switching to intermediate and late $M$ dwarfs and their single, discrete flares, it may on the other hand be possible to pin down the true basal coronal flux as observed on the Sun during quiescence, which seems to approach solar coronal conditions for these less active stars. The smooth transition in X-ray luminosity, flare rate, coronal temperatures and abundances we observe in our sequence of very active early $M$ dwarfs towards intermediate and late $\mathrm{M}$ dwarfs, i.e., from stars with radiative interiors to fully convective interiors, suggests that the different dynamo mechanisms thought to operate in these stars to not lead to easily observable consequences in their coronal properties.

\section{Summary and conclusions}

We have investigated the coronal properties of the M3.5/M4.5 EQ Peg binary system from their Chandra HETGS spectra. No large flares occur during our observations, and we find coronal densities ranging from $4 \times 10^{10} \mathrm{~cm}^{-3}$ to $3 \times 10^{11} \mathrm{~cm}^{-3}$ or consistent with the low-density limit from the O VII and Ne IX triplets, i.e. values typical for active stars. The ratio of $\mathrm{X}$-ray luminosities is 6:1 for EQ Peg A and B, but in terms of $L_{\mathrm{X}} / L_{\text {bol }}$, both stars are saturated. The differential emission measures of both components peak around $3 \mathrm{MK}$, and their abundances are similar and follow the inverse FIP effect.

We compared all seven $M$ dwarfs observed so far with the Chandra HETGS with two methods also suitable for spectra with low signal. The slope of the cumulative spectrum, which traces the continuum level and therefore the average coronal temperature, is very similar for spectral types M0.5 to M4 and then decreases. Emission measure-independent abundance ratios based on the line fluxes of strong lines with a similar temperature dependence confirm the existence of abundance anomalies compared to the solar photosphere for all $\mathrm{M}$ dwarfs in our sample. The ratios sensitive to a FIP bias, i.e. $\mathrm{Mg} / \mathrm{Ne}$ and $\mathrm{Ne} / \mathrm{Fe}$, show a clear trend with increasing spectral type to approach the solar photospheric level, while ratios insensitive to a FIP bias like $\mathrm{Si} / \mathrm{Mg}$ and $\mathrm{Ne} / \mathrm{O}$ stay at a constant level. These trends seem to be independent of the age of the stars.

Acknowledgements. We thank our referee, Prof. Carole Jordan, for constructive comments that helped to improve the paper. C.L. acknowledges financial support by the DLR under 50OR0105. J.-U.N. gratefully acknowledges support provided by NASA through Chandra Postdoctoral Fellowship grant PF5-60039 awarded by the Chandra X-ray Center, which is operated by the Smithsonian Astrophysical Observatory for NASA under contract NAS8-03060. This research made use of the SIMBAD database, operated at CDS, Strasbourg, France. CHIANTI is a collaborative project involving the NRL (USA), RAL (UK), MSSL (UK), the Universities of Florence (Italy) and Cambridge (UK), and George Mason University (USA). 


\section{References}

Acton, L. W., Catura, R. C., \& Joki, E. G. 1975, ApJ, 195, L93

Asplund, M., Grevesse, N., \& Sauval, A. J. 2005, in Cosmic Abundances as Records of Stellar Evolution and Nucleosynthesis, ASP Conf. Ser., 336, 25

Benedict, G. F., McArthur, B., Nelan, E., et al. 1998, AJ, 116, 429

Brosius, J. W., Davila, J. M., Thomas, R. J., \& Monsignori-Fossi, B. C. 1996, ApJS, 106, 143

Browning, M. K. 2008, ApJ, 676, 1262

Chabrier, G., \& Baraffe, I. 1997, A\&A, 327, 1039

Chabrier, G., \& Küker, M. 2006, A\&A, 446, 1027

Delfosse, X., Forveille, T., Perrier, C., \& Mayor, M. 1998, A\&A, 331, 581

Dobler, W., Stix, M., \& Brandenburg, A. 2006, ApJ, 638, 336

Drake, J. J., \& Testa, P. 2005, Nature, 436, 525

Durney, B. R., De Young, D. S., \& Roxburgh, I. W. 1993, Sol. Phys., 145, 207

Favata, F., Micela, G., Sciortino, S., \& Barbera, M. 1994, in Frontiers of Space and Ground-Based Astronomy, ed. W. Wamsteker, M. S. Longair, \& Y. Kondo, Astrophysics and Space Science Library, 187, 589

Favata, F., Reale, F., Micela, G., et al. 2000, A\&A, 353, 987

Feldman, U., \& Laming, J. M. 2000, Phys. Scr., 61, 222

Fleming, T. A., Giampapa, M. S., Schmitt, J. H. M. M., \& Bookbinder, J. A. 1993, ApJ, 410, 387

Fuhrmeister, B., Liefke, C., \& Schmitt, J. H. M. M. 2007, A\&A, 468, 221

Güdel, M., Audard, M., Magee, H., et al. 2001, A\&A, 365, L344

Güdel, M., Audard, M., Skinner, S. L., \& Horvath, M. I. 2002a, ApJ, 580, L73

Güdel, M., Audard, M., Sres, A., et al. 2002b, in Stellar Coronae in the Chandra and XMM-NEWTON Era, ed. F. Favata, \& J. J. Drake, ASP Conf. Ser., 277, 497

Güdel, M., Audard, M., Reale, F., Skinner, S. L., \& Linsky, J. L. 2004, A\&A, 416, 713

Haisch, B. M., Butler, C. J., Doyle, J. G., \& Rodono, M. 1987, A\&A, 181, 96

Hawley, S. L., Allred, J. C., Johns-Krull, C. M., et al. 2003, ApJ, 597, 535

Jackson, P. D., Kundu, M. R., \& White, S. M. 1989, A\&A, 210, 284

Katsova, M. M., Livshits, M. A., \& Schmitt, J. H. M. M. 2002, in Stellar Coronae in the Chandra and XMM-NEWTON Era, ed. F. Favata, \& J. J. Drake, ASP Conf. Ser., 277, 515

Kenyon, S. J., \& Hartmann, L. 1995, ApJS, 101, 117

Kron, G. E. 1952, ApJ, 115, 301

Lacy, C. H., Moffett, T. J., \& Evans, D. S. 1976, ApJS, 30, 85

Landi, E., Del Zanna, G., Young, P. R., et al. 2006, ApJS, 162, 261

Liefke, C., \& Schmitt, J. H. M. M. 2006, A\&A, 458, L1

Liefke, C., \& Schmitt, J. H. M. M., in prep.

Liefke, C., Reiners, A., \& Schmitt, J. H. M. M. 2007, Mem. Soc. Astron. Ital., 78,258

Liefke, C., Fuhrmeister, B., \& Schmitt, J. H. M. M., in prep.

Linsky, J. L., Ayres, T. R., Brown, A., \& Osten, R. A. 2002, Astron. Nachr., 323, 321

Magee, H. R. M., Güdel, M., Audard, M., \& Mewe, R. 2003, Adv. Space Res., 32, 1149

Maggio, A., \& Ness, J.-U. 2005, ApJ, 622, L57

Maggio, A., Drake, J. J., Kashyap, V., et al. 2004, ApJ, 613, 548

Mahmoud, F. M. 1993, Ap\&SS, 209, 237

Mazzotta, P., Mazzitelli, G., Colafrancesco, S., \& Vittorio, N. 1998, A\&AS, 133, 403

Mitra-Kraev, U., \& Ness, J.-U. 2006, in High Resolution X-ray Spectroscopy: towards XEUS and Con-X

Moffatt, H. K. 1978, Magnetic field generation in electrically conducting fluids (Cambridge, England: Cambridge University Press), 353
Monsignori Fossi, B. C., Landini, M., Fruscione, A., \& Dupuis, J. 1995, ApJ, 449,376

Mullan, D. J., \& MacDonald, J. 2001, ApJ, 559, 353

Ness, J.-U., \& Jordan, C. 2008, MNRAS, 385, 1691

Ness, J.-U., \& Wichmann, R. 2002, Astron. Nachr., 323, 129

Ness, J.-U., Schmitt, J. H. M. M., Burwitz, V., Mewe, R., \& Predehl, P. 2002, A\&A, 387, 1032

Ness, J.-U., Audard, M., Schmitt, J. H. M. M., \& Güdel, M. 2003a, Adv. Space Res., 32, 937

Ness, J.-U., Brickhouse, N. S., Drake, J. J., \& Huenemoerder, D. P. 2003b, ApJ, 598,1277

Ness, J.-U., Schmitt, J. H. M. M., Audard, M., Güdel, M., \& Mewe, R. 2003c, A\&A, 407, 347

Ness, J.-U., Güdel, M., Schmitt, J. H. M. M., Audard, M., \& Telleschi, A. 2004, A\&A, 427, 667

Nordon, R., \& Behar, E. 2007, A\&A, 464, 309

Nordon, R., \& Behar, E. 2008, A\&A, 482, 639

Norton, A. J., Wheatley, P. J., West, R. G., et al. 2007, A\&A, 467, 785

Osten, R. A., Hawley, S. L., Allred, J. C., Johns-Krull, C. M., \& Roark, C. 2005, ApJ, 621, 398

Osten, R. A., Hawley, S. L., Allred, J., et al. 2006, ApJ, 647, 1349

Pallavicini, R., Kundu, M. R., \& Jackson, P. D. 1986, in Lecture Notes in Physics, Cool Stars, Stellar Systems and the Sun (Berlin: Springer Verlag), ed. M. Zeilik, \& D. M. Gibson, 254, 225

Pallavicini, R., Tagliaferri, G., \& Stella, L. 1990, A\&A, 228, 403

Parker, E. N. 1955, ApJ, 122, 293

Parkinson, J. H. 1977, A\&A, 57, 185

Parsamyan, E. S. 1995, Astrophysics, 38, 206

Pettersen, B. R. 1976, Institute of Theoretical Astrophysics Blindern Oslo Reports, 46, 1

Pettersen, B. R., Olah, K., \& Sandmann, W. H. 1992, A\&AS, 96, 497

Pizzolato, N., Maggio, A., Micela, G., Sciortino, S., \& Ventura, P. 2003, A\&A, 397, 147

Raassen, A. J. J., Ness, J.-U., Mewe, R., et al. 2003, A\&A, 400, 671

Reiners, A. 2007, A\&A, 467, 259

Robrade, J., \& Schmitt, J. H. M. M. 2005, A\&A, 435, 1073

Robrade, J., Ness, J.-U., \& Schmitt, J. H. M. M. 2004, A\&A, 413, 317

Rutledge, R. E., Basri, G., Martín, E. L., \& Bildsten, L. 2000, ApJ, 538, L141

Sanz-Forcada, J., Favata, F., \& Micela, G. 2004, A\&A, 416, 281

Schmitt, J. H. M. M., \& Liefke, C. 2002, A\&A, 382, L9

Schmitt, J. H. M. M., \& Ness, J.-U. 2002, A\&A, 388, L13

Schmitt, J. H. M. M., \& Ness, J.-U. 2004, A\&A, 415, 1099

Schmitt, J. H. M. M., Reale, F., Liefke, C., et al. 2008, A\&A, 481, 799

Spiesman, W. J., \& Hawley, S. L. 1986, AJ, 92, 664

Stelzer, B., Burwitz, V., Audard, M., et al. 2002, A\&A, 392, 585

Stelzer, B., Schmitt, J. H. M. M., Micela, G., \& Liefke, C. 2006, A\&A, 460, L35

Telleschi, A., Güdel, M., Briggs, K., et al. 2005, ApJ, 622, 653

Testa, P., Drake, J. J., \& Peres, G. 2004, ApJ, 617, 508

Testa, P., Drake, J. J., Peres, G., \& Huenemoerder, D. P. 2007, ApJ, 665, 1349

Torres, C. A. O., Ferraz Mello, S., \& Quast, G. R. 1972, Astrophys. Lett., 11, 13

Torres, G., \& Ribas, I. 2002, ApJ, 567, 1140

Vaiana, G. S., Maggio, A., Micela, G., \& Sciortino, S. 1992, Mem. Soc. Astron. Ital., 63, 545

van den Besselaar, E. J. M., Raassen, A. J. J., Mewe, R., et al. 2003, A\&A, 411, 587

Yildiz, M. 2007, MNRAS, 374, 1264

Zboril, M., \& Byrne, P. B. 1998, MNRAS, 299, 753

Zuckerman, B., Song, I., Bessell, M. S., \& Webb, R. A. 2001, ApJ, 562, L87 